\title{
Hybrid Viscosity Approaches to General Systems of Variational Inequalities with Hierarchical Fixed Point Problem Constraints in Banach Spaces
}

\author{
Lu-Chuan Ceng, ${ }^{1}$ Saleh A. Al-Mezel, ${ }^{2}$ and Abdul Latif ${ }^{2}$ \\ ${ }^{1}$ Department of Mathematics, Shanghai Normal University and Scientific Computing Key Laboratory of Shanghai Universities, \\ Shanghai 200234, China \\ ${ }^{2}$ Department of Mathematics, King Abdulaziz University, P.O. Box 80203, Jeddah 21589, Saudi Arabia
}

Correspondence should be addressed to Saleh A. Al-Mezel; mathsaleh@yahoo.com

Received 4 August 2013; Revised 4 December 2013; Accepted 20 December 2013; Published 17 February 2014

Academic Editor: Hichem Ben-El-Mechaiekh

Copyright (C) $2014 \mathrm{Lu}$-Chuan Ceng et al. This is an open access article distributed under the Creative Commons Attribution License, which permits unrestricted use, distribution, and reproduction in any medium, provided the original work is properly cited.

\begin{abstract}
The purpose of this paper is to introduce and analyze hybrid viscosity methods for a general system of variational inequalities (GSVI) with hierarchical fixed point problem constraint in the setting of real uniformly convex and 2-uniformly smooth Banach spaces. Here, the hybrid viscosity methods are based on Korpelevich's extragradient method, viscosity approximation method, and hybrid steepest-descent method. We propose and consider hybrid implicit and explicit viscosity iterative algorithms for solving the GSVI with hierarchical fixed point problem constraint not only for a nonexpansive mapping but also for a countable family of nonexpansive mappings in $X$, respectively. We derive some strong convergence theorems under appropriate conditions. Our results extend, improve, supplement, and develop the recent results announced by many authors.
\end{abstract}

\section{Introduction}

Let $X$ be a real Banach space whose dual space is denoted by $X^{*}$. Let $U=\{x \in X:\|x\|=1\}$ denote the unit sphere of $X$. A Banach space $X$ is said to be uniformly convex if, for each $\epsilon \in(0,2]$, there exists $\delta>0$ such that, for all $x, y \in U$,

$$
\|x-y\| \geq \epsilon \Longrightarrow \frac{\|x+y\|}{2} \leq 1-\delta .
$$

It is known that a uniformly convex Banach space is reflexive and strictly convex. The normalized duality mapping $J$ : $X \rightarrow 2^{X^{*}}$ is defined by

$$
J(x)=\left\{x^{*} \in X^{*}:\left\langle x, x^{*}\right\rangle=\|x\|^{2}=\left\|x^{*}\right\|^{2}\right\}, \quad \forall x \in X,
$$

where $\langle\cdot, \cdot\rangle$ denotes the generalized duality pairing. It is an immediate consequence of the Hahn-Banach theorem that $J(x)$ is nonempty for each $x \in X$.

Let $C$ be a nonempty closed convex subset of a real Banach space $X$. A mapping $T: C \rightarrow C$ is said to be $L$-Lipschitzian if there exists a constant $L>0$ such that $\|T x-T y\| \leq$ $L\|x-y\|$ for all $x, y \in C$. In particular, if $L=1$, then $T$ is said to be nonexpansive. The set of fixed points of $T$ is denoted by $\operatorname{Fix}(T)$. We use the notation $\rightarrow$ to indicate the weak convergence and the one $\rightarrow$ to indicate the strong convergence. A mapping $A: C \rightarrow X$ is said to be

(i) accretive if, for each $x, y \in C$, there exists $j(x-y) \in$ $J(x-y)$ such that

$$
\langle A x-A y, j(x-y)\rangle \geq 0,
$$

where $J$ is the normalized duality mapping of $X$,

(ii) $\alpha$-inverse-strongly accretive if, for each $x, y \in C$, there exists $j(x-y) \in J(x-y)$ such that

$$
\langle A x-A y, j(x-y)\rangle \geq \alpha\|x-y\|^{2},
$$

for some $\alpha \in(0,1)$,

(iii) pseudocontractive if, for each $x, y \in C$, there exists $j(x-y) \in J(x-y)$ such that

$$
\langle A x-A y, j(x-y)\rangle \leq\|x-y\|^{2},
$$


(iv) $\beta$-strongly pseudocontractive if, for each $x, y \in C$, there exists $j(x-y) \in J(x-y)$ such that

$$
\langle A x-A y, j(x-y)\rangle \leq \beta\|x-y\|^{2}
$$

for some $\beta \in(0,1)$,

(v) $\lambda$-strictly pseudocontractive if, for each $x, y \in C$, there exists $j(x-y) \in J(x-y)$ such that

$$
\langle A x-A y, j(x-y)\rangle \leq\|x-y\|^{2}-\lambda\|x-y-(A x-A y)\|^{2}
$$

for some $\lambda \in(0,1)$.

It is worth emphasizing that the definition of the inversestrongly accretive mapping is based on that of the inversestrongly monotone mapping, which was studied by so many authors; see, for example, [1-7].

A Banach space $X$ is said to be smooth if the limit

$$
\lim _{t \rightarrow 0} \frac{\|x+t y\|-\|x\|}{t}
$$

exists for all $x, y \in X$; in this case, $X$ is also said to have a Gateaux differentiable norm. Moreover, it is said to be uniformly smooth if this limit is attained uniformly for $x, y \in U$; in this case, $X$ is also said to have a uniformly Frechet differentiable norm. The norm of $X$ is said to be the Frechet differential if, for each $x \in U$, this limit is attained uniformly for $y \in U$. In the meantime, we define a function $\rho:[0, \infty) \rightarrow[0, \infty)$ called the modulus of smoothness of $X$ as follows:

$$
\begin{gathered}
\rho(\tau)=\sup \left\{\frac{1}{2}(\|x+y\|+\|x-y\|)-1: x, y \in X,\right. \\
\|x\|=1,\|y\|=\tau\} .
\end{gathered}
$$

It is known that $X$ is uniformly smooth if and only if $\lim _{\tau \rightarrow 0} \rho(\tau) / \tau=0$. Let $q$ be a fixed real number with $1<q \leq$ 2. Then a Banach space $X$ is said to be $q$-uniformly smooth if there exists a constant $c>0$ such that $\rho(\tau) \leq c \tau^{q}$, for all $\tau>0$. As pointed out in [8], no Banach space is $q$-uniformly smooth for $q>2$. In addition, it is also known that $J$ is single-valued if and only if $X$ is smooth, whereas, if $X$ is uniformly smooth, then the mapping $J$ is norm-to-norm uniformly continuous on bounded subsets of $X$.

In a real smooth Banach space $X$, we say that an operator $A$ is strongly positive (see [9]), if there exists a constant $\bar{\gamma}>0$ with the property

$$
\begin{gathered}
\langle A x, J(x)\rangle \geq \bar{\gamma}\|x\|^{2}, \\
\|a I-b A\|=\sup _{\|x\| \leq 1}|\langle(a I-b A) x, J(x)\rangle|, \\
a \in[0,1], b \in[-1,1],
\end{gathered}
$$

where $I$ is the identity mapping.

Proposition CB (see [9, Lemma 2.5]). Let C be a nonempty closed convex subset of a uniformly smooth Banach space X. Let
$T: C \rightarrow C$ be a continuous pseudocontractive mapping with $\operatorname{Fix}(T) \neq \emptyset$ and let $f: C \rightarrow C$ be a fixed Lipschitzian strongly pseudocontractive mapping with pseudocontractive coefficient $\beta \in(0,1)$ and Lipschitzian constant $L>0$. Let $A: C \rightarrow C$ be a strongly positive linear bounded operator with coefficient $\bar{\gamma}>0$. Assume that $C \pm C \subset C$ and $0<\beta<\bar{\gamma}$. Let $\left\{x_{t}\right\}$ be defined by

$$
x_{t}=t f\left(x_{t}\right)+(I-t A) T x_{t} .
$$

Then, as $t \rightarrow 0,\left\{x_{t}\right\}$ converges strongly to some fixed point $p$ of $T$ such that $p$ is the unique solution in $\operatorname{Fix}(T)$ to the VIP:

$$
\langle(A-f) p, J(p-u)\rangle \leq 0, \quad \forall u \in \operatorname{Fix}(T) .
$$

On the other hand, Cai and $\mathrm{Bu}[10]$ considered the following general system of variational inequalities (GSVI) in a real smooth Banach space $X$, which involves finding $\left(x^{*}, y^{*}\right) \in C \times C$ such that

$$
\begin{aligned}
& \left\langle\mu_{1} B_{1} y^{*}+x^{*}-y^{*}, J\left(x-x^{*}\right)\right\rangle \geq 0, \quad \forall x \in C, \\
& \left\langle\mu_{2} B_{2} x^{*}+y^{*}-x^{*}, J\left(x-y^{*}\right)\right\rangle \geq 0, \quad \forall x \in C,
\end{aligned}
$$

where $C$ is a nonempty, closed, and convex subset of $X$, $B_{1}, B_{2}: C \rightarrow X$ are two nonlinear mappings, and $\mu_{1}$ and $\mu_{2}$ are two positive constants. Here the set of solutions of GSVI (13) is denoted by $\operatorname{GSVI}\left(C, B_{1}, B_{2}\right)$. Very recently, Cai and $\mathrm{Bu}[10]$ constructed an iterative algorithm for solving GSVI (13) and a common fixed point problem of an infinite family of nonexpansive mappings in a uniformly convex and 2-uniformly smooth Banach space. They proved the strong convergence of the proposed algorithm by virtue of the following inequality in a 2-uniformly smooth Banach space $X$.

Lemma 1 (see [11]). Let $X$ be a 2-uniformly smooth Banach space. Then, there exists a best smooth constant $\kappa>0$ such that

$$
\|x+y\|^{2} \leq\|x\|^{2}+2\langle y, J(x)\rangle+2\|\kappa y\|, \quad \forall x, y \in X,
$$

where $J$ is the normalized duality mapping from $X$ into $X^{*}$.

The authors [10] have used the following inequality in a real smooth and uniform convex Banach space $X$.

Proposition 2 (see [12]). Let $X$ be a real smooth and uniform convex Banach space and let $r>0$. Then, there exists a strictly increasing, continuous, and convex function $g:[0,2 r] \rightarrow \mathbf{R}$, $g(0)=0$ such that

$$
g(\|x-y\|) \leq\|x\|^{2}-2\langle x, J(y)\rangle+\|y\|^{2}, \quad \forall x, y \in B_{r},
$$

where $B_{r}=\{x \in X:\|x\| \leq r\}$.

\section{Preliminaries}

We list some lemmas that will be used in the sequel. Lemma 3 can be found in [13]. Lemma 4 is an immediate consequence of the subdifferential inequality of the function $(1 / 2)\|\cdot\|^{2}$. 
Lemma 3. Let $\left\{a_{n}\right\}$ be a sequence of nonnegative real numbers such that

$$
a_{n+1} \leq\left(1-b_{n}\right) a_{n}+b_{n} c_{n}, \quad \forall n \geq 0,
$$

where $\left\{b_{n}\right\}$ and $\left\{c_{n}\right\}$ are sequences of real numbers satisfying the following conditions:

(i) $\left\{b_{n}\right\} \subset[0,1]$ and $\sum_{n=0}^{\infty} b_{n}=\infty$;

(ii) either $\limsup _{n \rightarrow \infty} \leq 0$ or $\sum_{n=0}^{\infty}\left|b_{n} c_{n}\right|<\infty$.

Then, $\lim _{n \rightarrow \infty} a_{n}=0$.

Lemma 4. In a smooth Banach space $X$, there holds the inequality

$$
\begin{aligned}
\|x\|^{2}+2\langle y, J(x)\rangle & \leq\|x+y\|^{2} \\
& \leq\|x\|^{2}+2\langle y, J(x+y)\rangle, \quad \forall x, y \in X,
\end{aligned}
$$

where $J$ is the normalized duality mapping of $X$.

Let $\mu$ be a mean if $\mu$ is a continuous linear functional on $l^{\infty}$ satisfying $\|\mu\|=1=\mu(1)$. Then, we know that $\mu$ is a mean on $\mathbf{N}$ if and only if

$$
\inf \left\{a_{n}: n \in \mathbf{N}\right\} \leq \mu(a) \leq \sup \left\{a_{n}: n \in \mathbf{N}\right\}
$$

for every $a=\left(a_{1}, a_{2}, \ldots\right) \in l^{\infty}$. According to time and circumstances, we use $\mu_{n}\left(a_{n}\right)$ instead of $\mu(a)$. A mean $\mu$ on $\mathbf{N}$ is called a Banach limit if and only if

$$
\mu_{n}\left(a_{n}\right)=\mu_{n}\left(a_{n+1}\right)
$$

for every $a=\left(a_{1}, a_{2}, \ldots\right) \in l^{\infty}$. We know that, if $\mu$ is a Banach limit, then

$$
\liminf _{n \rightarrow \infty} a_{n} \leq \mu_{n}\left(a_{n}\right) \leq \limsup _{n \rightarrow \infty} a_{n}
$$

for every $a=\left(a_{1}, a_{2}, \ldots\right) \in l^{\infty}$. So, if $a=\left(a_{1}, a_{2}, \ldots\right), b=$ $\left(b_{1}, b_{2}, \ldots\right) \in l^{\infty}$, and $a_{n} \rightarrow c$ (resp., $\left.a_{n}-b_{n} \rightarrow 0\right)$, as $n \rightarrow \infty$, we have

$$
\mu_{n}\left(a_{n}\right)=\mu(a)=c \quad\left(\text { resp., } \mu_{n}\left(a_{n}\right)=\mu_{n}\left(b_{n}\right)\right) .
$$

Further, it is well known that there holds the following result.

Lemma 5 (see [14]). Let $C$ be a nonempty closed convex subset of a uniformly smooth Banach space X. Let $\left\{x_{n}\right\}$ be a bounded sequence of $X$; let $\mu$ be a mean on $\mathbf{N}$ and let $z \in C$. Then,

$$
\mu_{n}\left\|x_{n}-z\right\|^{2}=\min _{y \in C} \mu_{n}\left\|x_{n}-y\right\|^{2}
$$

if and only if

$$
\mu_{n}\left\langle y-z, J\left(x_{n}-z\right)\right\rangle \leq 0, \quad \forall y \in C,
$$

where $J$ is the normalized duality mapping of $X$.
Lemma 6 (see [9, Lemma 2.6]). Let $C$ be a nonempty closed convex subset of a real Banach space $X$ which has uniformly Gateaux differentiable norm. Let $T: C \rightarrow C$ be a continuous pseudocontractive mapping with $\operatorname{Fix}(T) \neq \emptyset$ and let $f: C \rightarrow$ $C$ be a fixed Lipschitzian strongly pseudocontractive mapping with pseudocontractive coefficient $\beta \in(0,1)$ and Lipschitzian constant $L>0$. Let $A: C \rightarrow C$ be a $\bar{\gamma}$-strongly positive linear bounded operator with coefficient $\bar{\gamma}>0$. Assume that $C \pm C \subset$ $C$ and that $\left\{x_{t}\right\}$ converges strongly to $p \in \operatorname{Fix}(T)$ as $t \rightarrow 0$, where $x_{t}$ is defined by $x_{t}=t f\left(x_{t}\right)+(I-t A) T x_{t}$. Suppose that $\left\{x_{n}\right\} \subset C$ is bounded and that $\lim _{n \rightarrow \infty}\left\|x_{n}-T x_{n}\right\|=0$. Then, $\lim \sup _{n \rightarrow \infty}\left\langle(f-A) p, J\left(x_{n}-p\right)\right\rangle \leq 0$.

Lemma 7. Let $C$ be a nonempty closed convex subset of a real smooth Banach space $X$. Let $F: C \rightarrow X$ be an $\alpha$-strongly accretive and $\lambda$-strictly pseudocontractive with $\alpha+\lambda \geq 1$. Then, $I-F$ is nonexpansive and $F$ is Lipschitz continuous with constant $(1+\sqrt{(1-\alpha) / \lambda})$. Further, for any fixed $\tau \in(0,1)$, I$\tau F$ is contractive with coefficient $1-\tau(1-\sqrt{(1-\alpha) / \lambda})$.

Proof. From the $\lambda$-strictly pseudocontractivity and $\alpha$ strongly accretivity of $F$, we have, for all $x, y \in C$,

$$
\begin{aligned}
& \lambda\|(I-F) x-(I-F) y\|^{2} \\
& \quad \leq\|x-y\|^{2}-\langle F(x)-F(y), J(x-y)\rangle \\
& \quad \leq(1-\alpha)\|x-y\|^{2},
\end{aligned}
$$

which implies that

$$
\|(I-F) x-(I-F) y\| \leq \sqrt{\frac{1-\alpha}{\lambda}}\|x-y\| .
$$

Because $\alpha+\lambda \geq 1 \Leftrightarrow \sqrt{(1-\alpha) / \lambda} \leq 1$, we know that $I-F$ is nonexpansive. Also note that

$$
\begin{aligned}
\|F(x)-F(y)\| & \leq\|(I-F) x-(I-F) y\|+\|x-y\| \\
& \leq\left(1+\sqrt{\frac{1-\alpha}{\lambda}}\right)\|x-y\| .
\end{aligned}
$$

Now, take a fixed $\tau \in(0,1)$ arbitrarily. Observe that, for all $x, y \in C$,

$$
\begin{aligned}
\|(I & -\tau F) x-(I-\tau F) y \| \\
& =\|(1-\tau)(x-y)+\tau[(I-F) x-(I-F) y]\| \\
& \leq(1-\tau)\|x-y\|+\tau\|(I-F) x-(I-F) y\| \\
& \leq(1-\tau)\|x-y\|+\tau\left(\sqrt{\frac{1-\alpha}{\lambda}}\right)\|x-y\| \\
& =\left(1-\tau\left(1-\sqrt{\frac{1-\alpha}{\lambda}}\right)\right)\|x-y\| .
\end{aligned}
$$

Because $\alpha+\lambda>1 \Leftrightarrow \sqrt{(1-\alpha) / \lambda}<1$, we know that $I-\tau F$ is contractive with coefficient $1-\tau(1-\sqrt{(1-\alpha) / \lambda})$. 
Let $D$ be a subset of $C$ and let $\Pi$ be a mapping of $C$ into $D$. Then, $\Pi$ is said to be sunny if

$$
\Pi[\Pi(x)+t(x-\Pi(x))]=\Pi(x),
$$

whenever $\Pi(x)+t(x-\Pi(x)) \in C$ for $x \in C$ and $t \geq 0$. A mapping $\Pi$ of $C$ into itself is called a retraction if $\Pi^{2}=\Pi$. If a mapping $\Pi$ of $C$ into itself is a retraction, then $\Pi(z)=z$ for every $z \in R(\Pi)$ where $R(\Pi)$ is the range of $\Pi$. A subset $D$ of $C$ is called a sunny nonexpansive retract of $C$ if there exists a sunny nonexpansive retraction from $C$ onto $D$. The following lemma concerns the sunny nonexpansive retraction.

Lemma 8 (see [15]). Let $C$ be a nonempty closed convex subset of a real smooth Banach space $X$. Let $D$ be a nonempty subset of $C$. Let $\Pi$ be a retraction of $C$ onto $D$. Then, the following are equivalent:

(i) $\Pi$ is sunny and nonexpansive;

(ii) $\|\Pi(x)-\Pi(y)\|^{2} \leq\langle x-y, J(\Pi(x)-\Pi(y))\rangle$, for all $x, y \in C$;

(iii) $\langle x-\Pi(x), J(y-\Pi(x))\rangle \leq 0$, for all $x \in C, y \in D$.

It is well known that, if $X=H$ is a Hilbert space, then a sunny nonexpansive retraction $\Pi_{C}$ is coincident with the metric projection from $X$ onto $C$; that is, $\Pi_{C}=P_{C}$. If $C$ is a nonempty closed convex subset of a strictly convex and uniformly smooth Banach space $X$ and if $T: C \rightarrow C$ is a nonexpansive mapping with the fixed point set $\operatorname{Fix}(T) \neq \emptyset$, then the set $\operatorname{Fix}(T)$ is a sunny nonexpansive retract of $C$.

Lemma 9. Let $C$ be a nonempty closed convex subset of a smooth Banach space $X$. Let $\Pi_{C}$ be a sunny nonexpansive retraction from $X$ onto $C$ and let $B_{1}, B_{2}: C \rightarrow X$ be nonlinear mappings. For given $x^{*}, y^{*} \in C,\left(x^{*}, y^{*}\right)$ is a solution of GSVI (13) if and only if $x^{*}=\Pi_{C}\left(y^{*}-\mu_{1} B_{1} y^{*}\right)$, where $y^{*}=$ $\Pi_{C}\left(x^{*}-\mu_{2} B_{2} x^{*}\right)$.

Proof. We can rewrite GSVI (13) as

$$
\begin{aligned}
& \left\langle x^{*}-\left(y^{*}-\mu_{1} B_{1} y^{*}\right), J\left(x-x^{*}\right)\right\rangle \geq 0, \quad \forall x \in C, \\
& \left\langle y^{*}-\left(x^{*}-\mu_{2} B_{2} x^{*}\right), J\left(x-y^{*}\right)\right\rangle \geq 0, \quad \forall x \in C,
\end{aligned}
$$

which is obviously equivalent to

$$
\begin{aligned}
& x^{*}=\Pi_{C}\left(y^{*}-\mu_{1} B_{1} y^{*}\right), \\
& y^{*}=\Pi_{C}\left(x^{*}-\mu_{2} B_{2} x^{*}\right),
\end{aligned}
$$

because of Lemma 8. This completes the proof. follows:

In terms of Lemma 9, define the mapping $G: C \rightarrow C$ as

$$
G(x):=\Pi_{C}\left(I-\mu_{1} B_{1}\right) \Pi_{C}\left(I-\mu_{2} B_{2}\right) x, \quad \forall x \in C .
$$

Then, we observe that

$$
x^{*}=\Pi_{C}\left[\Pi_{C}\left(x^{*}-\mu_{2} B_{2} x^{*}\right)-\mu_{1} B_{1} \Pi_{C}\left(x^{*}-\mu_{2} B_{2} x^{*}\right)\right],
$$

which implies that $x^{*}$ is a fixed point of the mapping $G$. Throughout this paper, the set of fixed points of the mapping $G$ is denoted by $\Omega$.

Lemma 10 (see [16]). Let $C$ be a nonempty closed convex subset of a strictly convex Banach space X. Let $\left\{T_{n}\right\}_{n=0}^{\infty}$ be a sequence of nonexpansive mappings on $C$. Suppose $\bigcap_{n=0}^{\infty} \operatorname{Fix}\left(T_{n}\right)$ is nonempty. Let $\left\{\lambda_{n}\right\}$ be a sequence of positive numbers with $\sum_{n=0}^{\infty} \lambda_{n}=1$. Then, a mapping $T$ on $C$ defined by $T x=\sum_{n=0}^{\infty} \lambda_{n} T_{n} x$ for $x \in C$ is well-defined, nonexpansive and $\operatorname{Fix}(T)=\bigcap_{n=0}^{\infty} \operatorname{Fix}\left(T_{n}\right)$ holds.

Lemma 11 (see [17]). Let $C$ be a nonempty closed convex subset of a Banach space $X$. Let $S_{1}, S_{2}, \ldots$ be a sequence of mappings of $C$ into itself. Suppose that $\sum_{n=1}^{\infty} \sup \left\{\left\|S_{n+1} x-S_{n} x\right\|: x \in C\right\}<$ $\infty$. Then, for each $y \in C,\left\{S_{n} y\right\}$ converges strongly to some point of $C$. Moreover, let $S$ be a mapping of $C$ into itself defined by $S y=\lim _{n \rightarrow \infty} S_{n} y$, for all $y \in C$. Then, $\lim _{n \rightarrow \infty} \sup \{\| S x-$ $\left.S_{n} x \|: x \in C\right\}=0$.

\section{GSVI with Hierarchical Fixed Point Problem Constraint for a Nonexpansive Mapping}

In this section, we introduce our hybrid implicit viscosity scheme for solving the GSVI (13) with hierarchical fixed point problem constraint for a nonexpansive mapping and show the strong convergence theorem. First, we list several useful and helpful lemmas.

Lemma 12 (see [10, Lemma 2.8]). Let $C$ be a nonempty closed convex subset of a real 2-uniformly smooth Banach space $X$. Let the mapping $B_{i}: C \rightarrow X$ be $\alpha_{i}$-inverse-strongly accretive. Then, one has

$$
\begin{aligned}
& \left\|\left(I-\mu_{i} B_{i}\right) x-\left(I-\mu_{i} B_{i}\right) y\right\|^{2} \\
& \quad \leq\|x-y\|^{2}+2 \mu_{i}\left(\mu_{i} \kappa^{2}-\alpha_{i}\right)\left\|B_{i} x-B_{i} y\right\|^{2}, \quad \forall x, y \in C,
\end{aligned}
$$

for $i=1,2$, where $\mu_{i}>0$. In particular, if $0<\mu_{i} \leq \alpha_{i} / \kappa^{2}$ (where $\kappa$ is the best constant of $X$ as in Lemma 1), then $I-\mu_{i} B_{i}$ is nonexpansive for $i=1,2$.

Lemma 13 (see [10, Lemma 2.9]). Let $C$ be a nonempty closed convex subset of a real 2-uniformly smooth Banach space $X$. Let $\Pi_{C}$ be a sunny nonexpansive retraction from $X$ onto $C$. Let the mapping $B_{i}: C \rightarrow X$ be $\alpha_{i}$-inverse-strongly accretive for $i=1,2$. Let $G: C \rightarrow C$ be the mapping defined by

$$
\begin{array}{r}
G x=\Pi_{C}\left[\Pi_{C}\left(x-\mu_{2} B_{2} x\right)-\mu_{1} B_{1} \Pi_{C}\left(x-\mu_{2} B_{2} x\right)\right], \\
\forall x \in C .
\end{array}
$$

If $0<\mu_{i} \leq \alpha_{i} / \kappa^{2}$, for $i=1,2$, then $G: C \rightarrow C$ is nonexpansive.

Lemma 14 (see [18]). Let $X$ be a Banach space, $C$ a nonempty closed and convex subset of $X$, and $T: C \rightarrow C$ a continuous 
and strong pseudocontraction. Then, T has a unique fixed point in $C$.

Lemma 15 (see [19]). Assume that $A$ is a strongly positive linear bounded operator on a smooth Banach space $X$ with coefficient $\bar{\gamma}>0$ and $0<\rho \leq\|A\|^{-1}$. Then, $\|I-\rho A\|^{2} \leq 1-\rho \bar{\gamma}$.

We now state and prove our first result.

Theorem 16. Let $C$ be a nonempty closed convex subset of a uniformly convex and 2-uniformly smooth Banach space $X$ such that $C \pm C \subset C$. Let $\Pi_{C}$ be a sunny nonexpansive retraction from $X$ onto $C$. Let the mapping $B_{i}: C \rightarrow X$ be $\alpha_{i}$-inverse-strongly accretive for $i=1,2$. Let $T: C \rightarrow C$ be a nonexpansive mapping such that $\Lambda=\operatorname{Fix}(T) \cap \Omega \neq \emptyset$ where $\Omega$ is the fixed point set of the mapping $G=\Pi_{C}(I-$ $\left.\mu_{1} B_{1}\right) \Pi_{C}\left(I-\mu_{2} B_{2}\right)$ with $0<\mu_{i}<\alpha_{i} / \kappa^{2}$ for $i=1,2$. Let $f: C \rightarrow C$ be a fixed Lipschitzian strongly pseudocontractive mapping with pseudocontractive coefficient $\beta \in(0,1)$ and Lipschitzian constant $L>0$, let $F: C \rightarrow C$ be $\alpha$-strongly accretive and $\lambda$-strictly pseudocontractive with $\alpha+\lambda>1$, and let $A: C \rightarrow C$ be a $\bar{\gamma}$-strongly positive linear bounded operator with $0<\bar{\gamma}-\beta \leq 1$. Let $\left\{x_{t}\right\}$ be defined by

$$
x_{t}=t f\left(x_{t}\right)+(I-t A)\left[G\left(T x_{t}\right)-\theta_{t} F G\left(T x_{t}\right)\right] \text {, }
$$

where $\left\{\theta_{t}: t \in(0,1)\right\} \subset[0,1)$ with $\lim _{t \rightarrow 0} \theta_{t} / t=0$. Then, as $t \rightarrow 0,\left\{x_{t}\right\}$ converges strongly to a point $p \in \Lambda$, which is the unique solution in $\Lambda$ to the VIP,

$$
\langle(A-f) p, J(p-u)\rangle \leq 0, \quad \forall u \in \Lambda .
$$

Proof. First, let us show that the net $\left\{x_{t}\right\}$ is defined well. As a matter of fact, define the mapping $S_{t}: C \rightarrow C$ as follows:

$$
S_{t} x=t f(x)+(I-t A)\left[G(T x)-\theta_{t} F G(T x)\right], \quad \forall x \in C
$$

We may assume, without loss of generality, that $t \leq\|A\|^{-1}$. Utilizing Lemmas 7, 13, and 15, we have

$$
\begin{aligned}
\left\langle S_{t} x-\right. & \left.S_{t} y, J(x-y)\right\rangle \\
= & t\langle f(x)-f(y), J(x-y)\rangle \\
& +\left\langle(I-t A)\left[\left(I-\theta_{t} F\right) G(T x)-\left(I-\theta_{t} F\right) G(T y)\right],\right. \\
& J(x-y)\rangle \\
\leq & t \beta\|x-y\|^{2}+(1-t \bar{\gamma}) \\
& \times\left\|\left(I-\theta_{t} F\right) G(T x)-\left(I-\theta_{t} F\right) G(T y)\right\|\|x-y\| \\
\leq & t \beta\|x-y\|^{2}+(1-t \bar{\gamma})\left(1-\theta_{t}\left(1-\sqrt{\frac{1-\alpha}{\lambda}}\right)\right) \\
& \times\|G(T x)-G(T y)\|\|x-y\| \\
\leq & t \beta\|x-y\|^{2}+(1-t \bar{\gamma})\|T x-T y\|\|x-y\|
\end{aligned}
$$

$$
\begin{aligned}
& \leq t \beta\|x-y\|^{2}+(1-t \bar{\gamma})\|x-y\|^{2} \\
& =(1-t(\bar{\gamma}-\beta))\|x-y\|^{2} .
\end{aligned}
$$

Hence, it is known that $S_{t}: C \rightarrow C$ is a continuous and strongly pseudocontractive mapping with pseudocontractive coefficient $1-t(\bar{\gamma}-\beta) \in(0,1)$ Thus, by Lemma 14 , we deduce that there exists a unique fixed point in $C$, denoted by $x_{t}$, which uniquely solves the fixed point equation

$$
x_{t}=t f\left(x_{t}\right)+(I-t A)\left[G\left(T x_{t}\right)-\theta_{t} F G\left(T x_{t}\right)\right] .
$$

Let us show the uniqueness of the solution of VIP (36). Suppose that both $p_{1} \in \Lambda$ and $p_{2} \in \Lambda$ are solutions to VIP (36). Then, we have

$$
\begin{aligned}
& \left\langle(A-f) p_{1}, J\left(p_{1}-p_{2}\right)\right\rangle \leq 0, \\
& \left\langle(A-f) p_{2}, J\left(p_{2}-p_{1}\right)\right\rangle \leq 0 .
\end{aligned}
$$

Adding up the above two inequalities, we obtain

$$
\left\langle(A-f) p_{1}-(A-f) p_{2}, J\left(p_{1}-p_{2}\right)\right\rangle \leq 0 .
$$

Note that

$$
\begin{aligned}
&\left\langle(A-f) p_{1}-(A-f) p_{2}, J\left(p_{1}-p_{2}\right)\right\rangle \\
&=\left\langle A\left(p_{1}-p_{2}\right), J\left(p_{1}-p_{2}\right)\right\rangle \\
&-\left\langle f\left(p_{1}\right)-f\left(p_{2}\right), J\left(p_{1}-p_{2}\right)\right\rangle \\
& \geq \bar{\gamma}\left\|p_{1}-p_{2}\right\|^{2}-\beta\left\|p_{1}-p_{2}\right\|^{2} \\
&=(\bar{\gamma}-\beta)\left\|p_{1}-p_{2}\right\|^{2} \geq 0 .
\end{aligned}
$$

Consequently, we have $p_{1}=p_{2}$, and the uniqueness is proved.

Next, let us show that, for some $a \in(0,1),\left\{x_{t}: t \in(0, a]\right\}$ is bounded. Indeed, since $\left\{\theta_{t}: t \in(0,1)\right\} \subset[0,1)$ with $\lim _{t \rightarrow 0}\left(\theta_{t} / t\right)=0$, there exists some $a \in(0,1)$ such that $0 \leq \theta_{t} / t<1$ for all $t \in(0, a]$. Take a fixed $p \in \operatorname{Fix}(\Lambda)$ arbitrarily. Utilizing Lemma 7 , we have

$$
\begin{aligned}
\left\|x_{t}-p\right\|^{2} & \\
= & \left\langle t\left(f\left(x_{t}\right)-f(p)\right)+(I-t A)\left[G\left(T x_{t}\right)-\theta_{t} F G\left(T x_{t}\right)-p\right]\right. \\
& \left.-t(A p-f(p)), J\left(x_{t}-p\right)\right\rangle \\
= & t\left\langle f\left(x_{t}\right)-f(p), J\left(x_{t}-p\right)\right\rangle \\
& +\left\langle(I-t A)\left[G\left(T x_{t}\right)-\theta_{t} F G\left(T x_{t}\right)-p\right], J\left(x_{t}-p\right)\right\rangle \\
& -t\left\langle(A-f) p, J\left(x_{t}-p\right)\right\rangle
\end{aligned}
$$




$$
\begin{aligned}
\leq & t \beta\left\|x_{t}-p\right\|^{2}+(1-t \bar{\gamma})\left\|G\left(T x_{t}\right)-\theta_{t} F G\left(T x_{t}\right)-p\right\| \\
& \times\left\|x_{t}-p\right\|+t\|(A-f) p\|\left\|x_{t}-p\right\| \\
\leq & t \beta\left\|x_{t}-p\right\|^{2}+(1-t \bar{\gamma}) \\
& \times\left[\left\|\left(I-\theta_{t} F\right) G\left(T x_{t}\right)-\left(I-\theta_{t} F\right) G(T p)\right\|\right. \\
& \left.\quad+\left\|\left(I-\theta_{t} F\right) G(T p)-p\right\|\right]\left\|x_{t}-p\right\| \\
& +t\|(A-f) p\|\left\|x_{t}-p\right\| \\
\leq & t \beta\left\|x_{t}-p\right\|^{2}+(1-t \bar{\gamma})\left(1-\theta_{t}\left(1-\sqrt{\frac{1-\alpha}{\lambda}}\right)\right) \\
& \times\left\|G\left(T x_{t}\right)-G(T p)\right\|\left\|x_{t}-p\right\| \\
& +(1-t \bar{\gamma}) \theta_{t}\|F p\|\left\|x_{t}-p\right\|+t\|(A-f) p\|\left\|x_{t}-p\right\| \\
\leq & t \beta\left\|x_{t}-p\right\|^{2}+(1-t \bar{\gamma})\left(1-\theta_{t}\left(1-\sqrt{\frac{1-\alpha}{\lambda}}\right)\right) \\
& \times\left\|T x_{t}-T p\right\|\left\|x_{t}-p\right\| \\
& +\theta_{t}\|F p\|\left\|x_{t}-p\right\|+t\|(A-f) p\|\left\|x_{t}-p\right\| \\
\leq & t \beta\left\|x_{t}-p\right\|^{2}+(1-t \bar{\gamma})\left\|x_{t}-p\right\|^{2} \\
& +\theta_{t}\|F p\|\left\|x_{t}-p\right\|+t\|(A-f) p\|\left\|x_{t}-p\right\| \\
= & (1-t(\bar{\gamma}-\beta))\left\|x_{t}-p\right\|^{2}+\theta_{t}\|F p\|\left\|x_{t}-p\right\| \\
& +t\|(A-f) p\|\left\|x_{t}-p\right\|,
\end{aligned}
$$

and, hence, for all $t \in(0, a]$,

$$
\begin{aligned}
\left\|x_{t}-p\right\| & \leq \frac{1}{\bar{\gamma}-\beta}\left(\|(A-f) p\|+\frac{\theta_{t}}{t}\|F p\|\right) \\
& \leq \frac{1}{\bar{\gamma}-\beta}(\|(A-f) p\|+\|F p\|) .
\end{aligned}
$$

Thus, this implies that $\left\{x_{t}: t \in(0, a]\right\}$ is bounded and so are $\left\{f\left(x_{t}\right): t \in(0, a]\right\},\left\{T x_{t}: t \in(0, a]\right\}$, and $\left\{G\left(T x_{t}\right): t \in(0, a]\right\}$.

Let us show that $\left\|T x_{t}-G\left(T x_{t}\right)\right\| \rightarrow 0$ as $t \rightarrow 0$.

Indeed, for simplicity, we put $q=\Pi_{C}\left(I-\mu_{2} B_{2}\right) p, \widehat{x}_{t}=T x_{t}$, $u_{t}=\Pi_{C}\left(I-\mu_{2} B_{2}\right) \hat{x}_{t}$, and $v_{t}=\Pi_{C}\left(I-\mu_{1} B_{1}\right) u_{t}$. Then, it is clear that $p=\Pi_{C}\left(I-\mu_{1} B_{1}\right) q$ and $v_{t}=G\left(\widehat{x}_{t}\right)=G\left(T x_{t}\right)$. Hence, from (43), it follows that

$$
\begin{aligned}
\left\|x_{t}-p\right\|^{2} & \\
\leq & t \beta\left\|x_{t}-p\right\|^{2}+(1-t \bar{\gamma})\left(1-\theta_{t}\left(1-\sqrt{\frac{1-\alpha}{\lambda}}\right)\right) \\
\quad & \left\|G\left(T x_{t}\right)-G(T p)\right\|\left\|x_{t}-p\right\| \\
& +(1-t \bar{\gamma}) \theta_{t}\|F p\|\left\|x_{t}-p\right\|+t\|(A-f) p\|\left\|x_{t}-p\right\|
\end{aligned}
$$

$$
\begin{aligned}
& \leq t \beta\left\|x_{t}-p\right\|^{2}+(1-t \bar{\gamma})\left\|v_{t}-p\right\|\left\|x_{t}-p\right\| \\
& \quad+\theta_{t}\|F p\|\left\|x_{t}-p\right\|+t\|(A-f) p\|\left\|x_{t}-p\right\| .
\end{aligned}
$$

From Lemma 12, we have

$$
\begin{aligned}
\left\|u_{t}-q\right\|^{2} & =\left\|\Pi_{C}\left(\widehat{x}_{t}-\mu_{2} B_{2} \widehat{x}_{t}\right)-\Pi_{C}\left(p-\mu_{2} B_{2} p\right)\right\|^{2} \\
& \leq\left\|\widehat{x}_{t}-p-\mu_{2}\left(B_{2} \widehat{x}_{t}-B_{2} p\right)\right\|^{2} \\
& \leq\left\|\widehat{x}_{t}-p\right\|^{2}-2 \mu_{2}\left(\alpha_{2}-\kappa^{2} \mu_{2}\right)\left\|B_{2} \widehat{x}_{t}-B_{2} p\right\|^{2}, \\
\left\|v_{t}-p\right\|^{2} & =\left\|\Pi_{C}\left(u_{t}-\mu_{1} B_{1} u_{t}\right)-\Pi_{C}\left(q-\mu_{1} B_{1} q\right)\right\|^{2} \\
& \leq\left\|u_{t}-q-\mu_{1}\left(B_{1} u_{t}-B_{1} q\right)\right\|^{2} \\
& \leq\left\|u_{t}-q\right\|^{2}-2 \mu_{1}\left(\alpha_{1}-\kappa^{2} \mu_{1}\right)\left\|B_{1} u_{t}-B_{1} q\right\|^{2} .
\end{aligned}
$$

From the last two inequalities, we obtain

$$
\begin{aligned}
\left\|v_{t}-p\right\|^{2} \leq & \left\|\widehat{x}_{t}-p\right\|^{2}-2 \mu_{2}\left(\alpha_{2}-\kappa^{2} \mu_{2}\right) \\
& \times\left\|B_{2} \widehat{x}_{t}-B_{2} p\right\|^{2}-2 \mu_{1}\left(\alpha_{1}-\kappa^{2} \mu_{1}\right) \\
& \times\left\|B_{1} u_{t}-B_{1} q\right\|^{2} \\
\leq & \left\|x_{t}-p\right\|^{2}-2 \mu_{2}\left(\alpha_{2}-\kappa^{2} \mu_{2}\right)\left\|B_{2} \widehat{x}_{t}-B_{2} p\right\|^{2} \\
& -2 \mu_{1}\left(\alpha_{1}-\kappa^{2} \mu_{1}\right)\left\|B_{1} u_{t}-B_{1} q\right\|^{2},
\end{aligned}
$$

which together with (45) implies that

$\left\|x_{t}-p\right\|^{2}$

$$
\begin{gathered}
\leq t \beta\left\|x_{t}-p\right\|^{2}+(1-t \bar{\gamma})\left\|v_{t}-p\right\|\left\|x_{t}-p\right\|+\theta_{t}\|F p\| \\
\times\left\|x_{t}-p\right\|+t\|(A-f) p\|\left\|x_{t}-p\right\| \\
\leq t \beta\left\|x_{t}-p\right\|^{2}+t\|(A-f) p\|\left\|x_{t}-p\right\|+(1-t \bar{\gamma}) \\
\times \frac{1}{2}\left(\left\|x_{t}-p\right\|^{2}+\left\|v_{t}-p\right\|^{2}\right)+\theta_{t}\|F p\|\left\|x_{t}-p\right\| \\
\leq t \beta\left\|x_{t}-p\right\|^{2}+t\|(A-f) p\|\left\|x_{t}-p\right\| \\
+(1-t \bar{\gamma}) \frac{1}{2}\left\{\left\|x_{t}-p\right\|^{2}+\left\|x_{t}-p\right\|^{2}\right. \\
-2 \mu_{2}\left(\alpha_{2}-\kappa^{2} \mu_{2}\right)\left\|B_{2} \widehat{x}_{t}-B_{2} p\right\|^{2} \\
-2 \mu_{1}\left(\alpha_{1}-\kappa^{2} \mu_{1}\right) \\
\left.\times\left\|B_{1} u_{t}-B_{1} q\right\|^{2}\right\}+\theta_{t}\|F p\|\left\|x_{t}-p\right\| \\
=(1-t(\bar{\gamma}-\beta))\left\|x_{t}-p\right\|^{2}+t\|(A-f) p\|\left\|x_{t}-p\right\| \\
-(1-t \bar{\gamma})\left[\mu_{2}\left(\alpha_{2}-\kappa^{2} \mu_{2}\right)\left\|B_{2} \widehat{x}_{t}-B_{2} p\right\|^{2}\right. \\
\left.+\mu_{1}\left(\alpha_{1}-\kappa^{2} \mu_{1}\right)\left\|B_{1} u_{t}-B_{1} q\right\|^{2}\right]
\end{gathered}
$$




$$
\begin{aligned}
& +\theta_{t}\|F p\|\left\|x_{t}-p\right\| \\
\leq & \left\|x_{t}-p\right\|^{2}+t\|(A-f) p\|\left\|x_{t}-p\right\|-(1-t \bar{\gamma}) \\
\times & {\left[\mu_{2}\left(\alpha_{2}-\kappa^{2} \mu_{2}\right)\left\|B_{2} \widehat{x}_{t}-B_{2} p\right\|^{2}\right.} \\
& \left.+\mu_{1}\left(\alpha_{1}-\kappa^{2} \mu_{1}\right)\left\|B_{1} u_{t}-B_{1} q\right\|^{2}\right]+\theta_{t}\|F p\|\left\|x_{t}-p\right\| .
\end{aligned}
$$

So, it immediately follows that

$$
\begin{gathered}
(1-t \bar{\gamma})\left[\mu_{2}\left(\alpha_{2}-\kappa^{2} \mu_{2}\right)\left\|B_{2} \widehat{x}_{t}-B_{2} p\right\|^{2}+\mu_{1}\left(\alpha_{1}-\kappa^{2} \mu_{1}\right)\right. \\
\left.\times\left\|B_{1} u_{t}-B_{1} q\right\|^{2}\right] \\
\leq t\|(A-f) p\|\left\|x_{t}-p\right\|+\theta_{t}\|F p\|\left\|x_{t}-p\right\| .
\end{gathered}
$$

Since $0<\mu_{i}<\alpha_{i} / \kappa^{2}$, for $i=1,2$, we have

$$
\lim _{t \rightarrow 0}\left\|B_{2} \widehat{x}_{t}-B_{2} p\right\|=0, \quad \lim _{t \rightarrow 0}\left\|B_{1} u_{t}-B_{1} q\right\|=0 .
$$

Utilizing Proposition 2 and Lemma 8, we have that there exists $g_{1}$ such that

$$
\begin{aligned}
\left\|u_{t}-q\right\|^{2}= & \left\|\Pi_{C}\left(\widehat{x}_{t}-\mu_{2} B_{2} \hat{x}_{t}\right)-\Pi_{C}\left(p-\mu_{2} B_{2} p\right)\right\|^{2} \\
\leq & \left\langle\hat{x}_{t}-\mu_{2} B_{2} \widehat{x}_{t}-\left(p-\mu_{2} B_{2} p\right), J\left(u_{t}-q\right)\right\rangle \\
= & \left\langle\widehat{x}_{t}-p, J\left(u_{t}-q\right)\right\rangle+\mu_{2}\left\langle B_{2} p-B_{2} \widehat{x}_{t}, J\left(u_{t}-q\right)\right\rangle \\
\leq & \frac{1}{2}\left[\left\|\widehat{x}_{t}-p\right\|^{2}+\left\|u_{t}-q\right\|^{2}\right. \\
& \left.\quad-g_{1}\left(\left\|\widehat{x}_{t}-u_{t}-(p-q)\right\|\right)\right] \\
& +\mu_{2}\left\|B_{2} p-B_{2} \hat{x}_{t}\right\|\left\|u_{t}-q\right\|,
\end{aligned}
$$

which implies that

$$
\begin{gathered}
\left\|u_{t}-q\right\|^{2} \leq\left\|\widehat{x}_{t}-p\right\|^{2}-g_{1}\left(\left\|\hat{x}_{t}-u_{t}-(p-q)\right\|\right) \\
+2 \mu_{2}\left\|B_{2} p-B_{2} \hat{x}_{t}\right\|\left\|u_{t}-q\right\| .
\end{gathered}
$$

In the same way, we derive that there exists $g_{2}$ :

$$
\begin{aligned}
\left\|v_{t}-p\right\|^{2}= & \left\|\Pi_{C}\left(u_{t}-\mu_{1} B_{1} u_{t}\right)-\Pi_{C}\left(q-\mu_{1} B_{1} q\right)\right\|^{2} \\
\leq & \left\langle u_{t}-\mu_{1} B_{1} u_{t}-\left(q-\mu_{1} B_{1} q\right), J\left(v_{t}-p\right)\right\rangle \\
= & \left\langle u_{t}-q, J\left(v_{t}-p\right)\right\rangle+\mu_{1}\left\langle B_{1} q-B_{1} u_{t}, J\left(v_{t}-p\right)\right\rangle \\
\leq & \frac{1}{2}\left[\left\|u_{t}-q\right\|^{2}+\left\|v_{t}-p\right\|^{2}\right. \\
& \left.\quad-g_{2}\left(\left\|u_{t}-v_{t}+(p-q)\right\|\right)\right] \\
& +\mu_{1}\left\|B_{1} q-B_{1} u_{t}\right\|\left\|v_{t}-p\right\|,
\end{aligned}
$$

which implies that

$$
\begin{aligned}
\left\|v_{t}-p\right\|^{2} \leq & \left\|u_{t}-q\right\|^{2}-g_{2}\left(\left\|u_{t}-v_{t}+(p-q)\right\|\right) \\
& +2 \mu_{1}\left\|B_{1} q-B_{1} u_{t}\right\|\left\|v_{t}-p\right\| .
\end{aligned}
$$

Substituting (52) for (54), we get

$$
\begin{aligned}
\left\|v_{t}-p\right\|^{2} \leq & \left\|\widehat{x}_{t}-p\right\|^{2}-g_{1}\left(\left\|\widehat{x}_{t}-u_{t}-(p-q)\right\|\right) \\
& -g_{2}\left(\left\|u_{t}-v_{t}+(p-q)\right\|\right) \\
& +2 \mu_{2}\left\|B_{2} p-B_{2} \widehat{x}_{t}\right\|\left\|u_{t}-q\right\| \\
& +2 \mu_{1}\left\|B_{1} q-B_{1} u_{t}\right\|\left\|v_{t}-p\right\| \\
\leq & \left\|x_{t}-p\right\|^{2}-g_{1}\left(\left\|\widehat{x}_{t}-u_{t}-(p-q)\right\|\right) \\
& -g_{2}\left(\left\|u_{t}-v_{t}+(p-q)\right\|\right) \\
& +2 \mu_{2}\left\|B_{2} p-B_{2} \widehat{x}_{t}\right\|\left\|u_{t}-q\right\| \\
& +2 \mu_{1}\left\|B_{1} q-B_{1} u_{t}\right\|\left\|v_{t}-p\right\|,
\end{aligned}
$$

which together with (45) implies that

$$
\begin{aligned}
\| x_{t}- & p \|^{2} \\
\leq & t \beta\left\|x_{t}-p\right\|^{2}+(1-t \bar{\gamma})\left\|v_{t}-p\right\|\left\|x_{t}-p\right\|+\theta_{t}\|F p\| \\
& \times\left\|x_{t}-p\right\|+t\|(A-f) p\|\left\|x_{t}-p\right\| \\
\leq & t \beta\left\|x_{t}-p\right\|^{2}+t\|(A-f) p\|\left\|x_{t}-p\right\|+(1-t \bar{\gamma}) \\
& \times \frac{1}{2}\left(\left\|x_{t}-p\right\|^{2}+\left\|v_{t}-p\right\|^{2}\right)+\theta_{t}\|F p\|\left\|x_{t}-p\right\| \\
\leq & t \beta\left\|x_{t}-p\right\|^{2}+t\|(A-f) p\|\left\|x_{t}-p\right\|+(1-t \bar{\gamma}) \\
& \times \frac{1}{2}\left\{\left\|x_{t}-p\right\|^{2}+\left\|x_{t}-p\right\|^{2}\right. \\
& -g_{1}\left(\left\|\widehat{x}_{t}-u_{t}-(p-q)\right\|\right) \\
& -g_{2}\left(\left\|u_{t}-v_{t}+(p-q)\right\|\right) \\
& +2 \mu_{2}\left\|B_{2} p-B_{2} \widehat{x}_{t}\right\|\left\|u_{t}-q\right\| \\
& \left.+2 \mu_{1}\left\|B_{1} q-B_{1} u_{t}\right\|\left\|v_{t}-p\right\|\right\} \\
& +\theta_{t}\|F p\|\left\|x_{t}-p\right\| \\
= & (1-t(\bar{\gamma}-\beta))\left\|x_{t}-p\right\|^{2}+t\|(A-f) p\|\left\|x_{t}-p\right\| \\
- & (1-t \bar{\gamma}) \\
\times & \frac{1}{2}\left[g_{1}\left(\left\|\widehat{x}_{t}-u_{t}-(p-q)\right\|\right)\right. \\
& \left.+g_{2}\left(\left\|u_{t}-v_{t}+(p-q)\right\|\right)\right]+(1-t \bar{\gamma}) \\
\times & {\left[\mu_{2}\left\|B_{2} p-B_{2} \widehat{x}_{t}\right\|\left\|u_{t}-q\right\|\right.} \\
& \left.+\mu_{1}\left\|B_{1} q-B_{1} u_{t}\right\|\left\|v_{t}-p\right\|\right]
\end{aligned}
$$




$$
\begin{aligned}
& +\theta_{t}\|F p\|\left\|x_{t}-p\right\| \\
\leq & \left\|x_{t}-p\right\|^{2}+t\|(A-f) p\|\left\|x_{t}-p\right\|-(1-t \bar{\gamma}) \\
& \times \frac{1}{2}\left[g_{1}\left(\left\|\widehat{x}_{t}-u_{t}-(p-q)\right\|\right)\right. \\
& \left.\quad+g_{2}\left(\left\|u_{t}-v_{t}+(p-q)\right\|\right)\right] \\
& +\mu_{2}\left\|B_{2} p-B_{2} \widehat{x}_{t}\right\|\left\|u_{t}-q\right\| \\
& +\mu_{1}\left\|B_{1} q-B_{1} u_{t}\right\|\left\|v_{t}-p\right\|+\theta_{t}\|F p\|\left\|x_{t}-p\right\| .
\end{aligned}
$$

So, it immediately follows that

$$
\begin{aligned}
(1-t \bar{\gamma}) \frac{1}{2}\left[g_{1}\left(\left\|\widehat{x}_{t}-u_{t}-(p-q)\right\|\right)\right. \\
\left.\quad+g_{2}\left(\left\|u_{t}-v_{t}+(p-q)\right\|\right)\right] \\
\leq t\|(A-f) p\|\left\|x_{t}-p\right\|+\mu_{2}\left\|B_{2} p-B_{2} \widehat{x}_{t}\right\|\left\|u_{t}-q\right\| \\
+\mu_{1}\left\|B_{1} q-B_{1} u_{t}\right\|\left\|v_{t}-p\right\|+\theta_{t}\|F p\|\left\|x_{t}-p\right\| .
\end{aligned}
$$

Hence, from (50), we conclude that

$$
\begin{aligned}
& \lim _{t \rightarrow 0} g_{1}\left(\left\|\widehat{x}_{t}-u_{t}-(p-q)\right\|\right)=0, \\
& \lim _{t \rightarrow 0} g_{2}\left(\left\|u_{t}-v_{t}+(p-q)\right\|\right)=0 .
\end{aligned}
$$

Utilizing the properties of $g_{1}$ and $g_{2}$, we get

$$
\begin{aligned}
& \lim _{t \rightarrow 0}\left\|\widehat{x}_{t}-u_{t}-(p-q)\right\|=0, \\
& \lim _{t \rightarrow 0}\left\|u_{t}-v_{t}+(p-q)\right\|=0,
\end{aligned}
$$

which leads to

$$
\begin{aligned}
\left\|\hat{x}_{t}-v_{t}\right\| \leq & \left\|\widehat{x}_{t}-u_{t}-(p-q)\right\| \\
& +\left\|u_{t}-v_{t}+(p-q)\right\| \longrightarrow 0 \quad \text { as } t \longrightarrow 0 .
\end{aligned}
$$

That is,

$$
\lim _{t \rightarrow 0}\left\|T x_{t}-G\left(T x_{t}\right)\right\|=\lim _{t \rightarrow 0}\left\|\widehat{x}_{t}-v_{t}\right\|=0 .
$$

Note that $\left\{x_{t}: t \in(0, a]\right\}$ is bounded and so are $\left\{f\left(x_{t}\right)\right.$ : $t \in(0, a]\},\left\{T x_{t}: t \in(0, a]\right\}$, and $\left\{G\left(T x_{t}\right): t \in(0, a]\right\}$. Hence, we have

$$
\begin{aligned}
& \left\|x_{t}-G\left(T x_{t}\right)\right\| \\
& =t\left\|f\left(x_{t}\right)-A G\left(T x_{t}\right)-\frac{\theta_{t}}{t}(I-t A) F G\left(T x_{t}\right)\right\| \longrightarrow 0,
\end{aligned}
$$

as $t \rightarrow 0$. Also, observe that

$$
\left\|x_{t}-T x_{t}\right\| \leq\left\|x_{t}-G\left(T x_{t}\right)\right\|+\left\|G\left(T x_{t}\right)-T x_{t}\right\| .
$$

This together with (61) and (62) implies that

$$
\lim _{t \rightarrow 0}\left\|x_{t}-T x_{t}\right\|=0
$$

Utilizing the nonexpansivity of $G$, we obtain

$$
\begin{aligned}
\left\|x_{t}-G x_{t}\right\| & \leq\left\|x_{t}-G\left(T x_{t}\right)\right\|+\left\|G\left(T x_{t}\right)-G x_{t}\right\| \\
& \leq\left\|x_{t}-G\left(T x_{t}\right)\right\|+\left\|T x_{t}-x_{t}\right\|,
\end{aligned}
$$

which together with (62) and (64) implies that

$$
\lim _{t \rightarrow 0}\left\|x_{t}-G x_{t}\right\|=0 \text {. }
$$

Now, let $\left\{t_{k}\right\}$ be a sequence in $(0, a]$ that converges to 0 as $k \rightarrow$ $\infty$, and define a function $g$ on $C$ by

$$
g(x)=\mu_{k} \frac{1}{2}\left\|x_{t_{k}}-x\right\|^{2}, \quad \forall x \in C,
$$

where $\mu$ is a Banach limit. Define the set

$$
K:=\{w \in C: g(w)=\min \{g(y): y \in C\}\}
$$

and the mapping

$$
W x=(1-\theta) T x+\theta G x, \quad \forall x \in C,
$$

where $\theta$ is a constant in $(0,1)$. Then, by Lemma 10 , we know that $\operatorname{Fix}(W)=\operatorname{Fix}(T) \cap \operatorname{Fix}(G)=\Lambda$. We observe that

$$
\begin{aligned}
\left\|x_{t}-W x_{t}\right\| & =\left\|(1-\theta)\left(x_{t}-T x_{t}\right)+\theta\left(x_{t}-G x_{t}\right)\right\| \\
& \leq(1-\theta)\left\|x_{t}-T x_{t}\right\|+\theta\left\|x_{t}-G x_{t}\right\| .
\end{aligned}
$$

So, from (64) and (66), we obtain

$$
\lim _{n \rightarrow \infty}\left\|x_{t}-W x_{t}\right\|=0
$$

Since $X$ is a uniformly smooth Banach space, $K$ is a nonempty bounded closed convex subset of $C$; for more details, see [14]. We claim that $K$ is also invariant under the nonexpansive mapping $W$. Indeed, noticing (71), we have, for $w \in K$,

$$
\begin{aligned}
g(W w) & =\mu_{k} \frac{1}{2}\left\|x_{t_{k}}-W w\right\|^{2}=\mu_{k} \frac{1}{2}\left\|W x_{t_{k}}-W w\right\|^{2} \\
& \leq \mu_{k} \frac{1}{2}\left\|x_{t_{k}}-w\right\|^{2}=g(w) .
\end{aligned}
$$

Since every nonempty closed bounded convex subset of a uniformly smooth Banach space $X$ has the fixed point property for nonexpansive mappings and $W$ is a nonexpansive mapping of $K, W$ has a fixed point in $K$, say $p$. Utilizing Lemma 5, we get

$$
\mu_{k}\left\langle x-p, J\left(x_{t_{k}}-p\right)\right\rangle \leq 0, \quad \forall x \in C .
$$

Putting $x=(f-A) p+p \in C$, we have

$$
\mu_{k}\left\langle(f-A) p, J\left(x_{t_{k}}-p\right)\right\rangle \leq 0, \quad \forall x \in C .
$$


Since $x_{t_{k}}-p=t_{k}\left(f\left(x_{t_{k}}\right)-f(p)\right)+\left(I-t_{k} A\right)\left[G\left(T x_{t_{k}}\right)-\theta_{t_{k}}\right.$ $\left.F G\left(T x_{t_{k}}\right)-p\right]-t_{k}(A-f) p$, we get

$$
\begin{aligned}
\| x_{t_{k}}- & p \|^{2} \\
= & t_{k}\left\langle f\left(x_{t_{k}}\right)-f(p), J\left(x_{t_{k}}-p\right)\right\rangle \\
& +\left\langle\left(I-t_{k} A\right)\left(G\left(T x_{t_{k}}\right)-p\right), J\left(x_{t_{k}}-p\right)\right\rangle \\
& -\theta_{t_{k}}\left\langle\left(I-t_{k} A\right) F G\left(T x_{t_{k}}\right), J\left(x_{t_{k}}-p\right)\right\rangle \\
& -t_{k}\left\langle(A-f) p, J\left(x_{t_{k}}-p\right)\right\rangle \\
\leq & t_{k} \beta\left\|x_{t_{k}}-p\right\|^{2}+t_{k}\left\langle(f-A) p, J\left(x_{t_{k}}-p\right)\right\rangle \\
& +\left(1-t_{k} \bar{\gamma}\right)\left\|G\left(T x_{t_{k}}\right)-p\right\|\left\|x_{t_{k}}-p\right\| \\
& +\left(1-t_{k} \bar{\gamma}\right) \theta_{t_{k}}\left\|F G\left(T x_{t_{k}}\right)\right\|\left\|x_{t_{k}}-p\right\| \\
\leq & t_{k} \beta\left\|x_{t_{k}}-p\right\|^{2}+t_{k}\left\langle(f-A) p, J\left(x_{t_{k}}-p\right)\right\rangle \\
& +\left(1-t_{k} \bar{\gamma}\right)\left\|x_{t_{k}}-p\right\|^{2}+\theta_{t_{k}}\left\|F G\left(T x_{t_{k}}\right)\right\|\left\|x_{t_{k}}-p\right\| .
\end{aligned}
$$

It follows that

$$
\begin{aligned}
\left\|x_{t_{k}}-p\right\|^{2} \leq \frac{1}{\bar{\gamma}-\beta}[ & \left\langle(f-A) p, J\left(x_{t_{k}}-p\right)\right\rangle \\
& \left.+\frac{\theta_{t_{k}}}{t_{k}}\left\|F G\left(T x_{t_{k}}\right)\right\|\left\|x_{t_{k}}-p\right\|\right] .
\end{aligned}
$$

Since $\lim _{k \rightarrow \infty}\left(\theta_{t_{k}} / t_{k}\right)=0$, from (74) and the boundedness of sequences $\left\{F G\left(T x_{t_{k}}\right)\right\},\left\{x_{t_{k}}\right\}$, it follows that

$$
\begin{aligned}
& \mu_{k}\left\|x_{t_{k}}-p\right\|^{2} \leq \frac{1}{\bar{\gamma}-\beta} \mu_{k}\left[\left\langle(f-A) p, J\left(x_{t_{k}}-p\right)\right\rangle\right. \\
&\left.+\frac{\theta_{t_{k}}}{t_{k}}\left\|F G\left(T x_{t_{k}}\right)\right\|\left\|x_{t_{k}}-p\right\|\right] \\
&=\frac{1}{\bar{\gamma}-\beta}\left[\mu_{k}\left\langle(f-A) p, J\left(x_{t_{k}}-p\right)\right\rangle\right. \\
&\left.+\mu_{k}\left(\frac{\theta_{t_{k}}}{t_{k}}\left\|F G\left(T x_{t_{k}}\right)\right\|\left\|x_{t_{k}}-p\right\|\right)\right] \leq 0 .
\end{aligned}
$$

Therefore, for the sequence $\left\{x_{t_{k}}\right\}$ in $\left\{x_{t}: t \in(0, a]\right\}$, there exists a subsequence which is still denoted by $\left\{x_{t_{k}}\right\}$ that converges strongly to some fixed point $p$ of $W$.

Now, we claim that such a $p$ is the unique solution in $\Lambda$ to the VIP (36).
Indeed, from (35), it follows that for all $u \in \Lambda=\operatorname{Fix}(T) \cap \Omega$

$$
\begin{array}{rl}
\left\|x_{t}-u\right\|^{2} & t\left\langle f\left(x_{t}\right)-f(u), J\left(x_{t}-u\right)\right\rangle \\
& +\left\langle(I-t A)\left[G\left(T x_{t}\right)-\theta_{t} F G\left(T x_{t}\right)-u\right], J\left(x_{t}-u\right)\right\rangle \\
& -t\left\langle(A-f) u, J\left(x_{t}-u\right)\right\rangle \\
= & \left\langle( I - t A ) \left[\left(I-\theta_{t} F\right) G\left(T x_{t}\right)-\left(I-\theta_{t} F\right) u\right.\right. \\
& \left.\left.\quad+\left(I-\theta_{t} F\right) u-u\right], J\left(x_{t}-u\right)\right\rangle \\
& +t\left\langle f\left(x_{t}\right)-f(u), J\left(x_{t}-u\right)\right\rangle-t\left\langle(A-f) u, J\left(x_{t}-u\right)\right\rangle \\
\leq & (1-t \bar{\gamma})\left[\left\|\left(I-\theta_{t} F\right) G\left(T x_{t}\right)-\left(I-\theta_{t} F\right) u\right\|\right. \\
& \left.\quad+\left\|\left(I-\theta_{t} F\right) u-u\right\|\right] \\
& \times\left\|x_{t}-u\right\|+t \beta\left\|x_{t}-u\right\|^{2}-t\left\langle(A-f) u, J\left(x_{t}-u\right)\right\rangle \\
\leq & (1-t \bar{\gamma})\left[\left(1-\theta_{t}\left(1-\sqrt{\frac{1-\alpha}{\lambda}}\right)\right)\left\|x_{t}-u\right\|+\theta_{t}\|F u\|\right] \\
& \times\left\|x_{t}-u\right\|+t \beta\left\|x_{t}-u\right\|^{2}-t\left\langle(A-f) u, J\left(x_{t}-u\right)\right\rangle \\
\leq & (1-t \bar{\gamma})\left[\left\|x_{t}-u\right\|+\theta_{t}\|F u\|\right]\left\|x_{t}-u\right\| \\
& +t \beta\left\|x_{t}-u\right\|^{2}-t\left\langle(A-f) u, J\left(x_{t}-u\right)\right\rangle \\
\leq & (1-t(\bar{\gamma}-\beta))\left\|x_{t}-u\right\|^{2}+\theta_{t}\|F u\| \\
& \times\left\|x_{t}-u\right\|-t\left\langle(A-f) u, J\left(x_{t}-u\right)\right\rangle \\
\leq & \left\|x_{t}-u\right\|^{2}+\theta_{t}\|F u\|\left\|x_{t}-u\right\|-t\left\langle(A-f) u, J\left(x_{t}-u\right)\right\rangle,
\end{array}
$$

which hence implies that

$$
\left\langle(A-f) u, J\left(x_{t}-u\right)\right\rangle \leq \frac{\theta_{t}}{t}\|F u\|\left\|x_{t}-u\right\|, \quad \forall u \in \Lambda .
$$

Since $x_{t_{k}} \rightarrow p$ as $t_{k} \rightarrow 0$ and $\lim _{t \rightarrow 0}\left(\theta_{t} / t\right)=0$, we obtain from the last inequality that

$$
\langle(A-f) u, J(p-u)\rangle \leq 0, \quad \forall u \in \Lambda .
$$

Utilizing the well-known Minty-type Lemma, we get

$$
\langle(A-f) p, J(p-u)\rangle \leq 0, \quad \forall u \in \Lambda .
$$

So, $p$ is a solution in $\Lambda$ to the VIP (36).

In order to prove that the net $\left\{x_{t}: t \in(0, a]\right\}$ converges strongly to $p$ as $t \rightarrow 0$, suppose that there exists another subsequence $\left\{x_{s_{k}}\right\} \subset\left\{x_{t}\right\}$ such that $x_{s_{k}} \rightarrow q$ as $s_{k} \rightarrow 0$; then we also have $q \in \operatorname{Fix}(W)=\operatorname{Fix}(T) \cap \Omega=: \Lambda$ due to (71). Repeating the same argument as above, we know that $q$ is another solution in $\Lambda$ to the VIP (36). In terms of the uniqueness of solutions in $\Lambda$ to the VIP (36), we immediately get $p=q$. This completes the proof. 
Remark 17. It is worth emphasizing that, in the assertion of Theorem 16, "as $t \rightarrow 0,\left\{x_{t}\right\}$ converges strongly to a point $p \in \Lambda$," this $p$ depends on no one of the mappings $f, A$, and $F$. Indeed, although $\left\{x_{t}\right\}$ is defined by

$$
x_{t}=t f\left(x_{t}\right)+(I-t A)\left[G\left(T x_{t}\right)-\theta_{t} F G\left(T x_{t}\right)\right], \quad \forall t \in(0,1),
$$

in the proof of Theorem 16, it can be readily seen that $p$ is first found out as a fixed point of the nonexpansive self-mapping $W$ of $K$. This shows that $p$ depends on no one of the mappings $f, A$, and $F$.

Remark 18. Theorem 16 improves, extends, supplements, and develops Cai and $\mathrm{Bu}$ [9, Lemma 2.5] in the following aspects.

(i) The GSVI (13) with hierarchical fixed point problem constraint for a nonexpansive mapping is more general and more subtle than the problem in Cai and $\mathrm{Bu}$ [9, Lemma 2.5] because our problem is to find a point $p \in \Lambda=\operatorname{Fix}(T) \cap \Omega$, which is the unique solution in $\Lambda$ to the VIP:

$$
\langle(A-f) p, J(p-u)\rangle \leq 0, \quad \forall u \in \Lambda .
$$

(ii) The iterative scheme in [9, Lemma 2.5] is extended to develop the iterative scheme in Theorem 16 by virtue of hybrid steepest-descent method. The iterative scheme in Theorem 16 is more advantageous and more flexible than the iterative scheme of [9, Lemma 2.5] because our iterative scheme involves solving two problems: the GSVI (13) and the fixed point problem of a nonexpansive mapping $T$.

(iii) The iterative scheme in Theorem 16 is very different from the iterative scheme in [9, Lemma 2.5] because our iterative scheme involves hybrid steepest-descent method (namely, we add a strongly accretive and strictly pseudocontractive mapping $F$ in our iterative scheme) and because the mapping $T$ in [9, Lemma 2.5] is replaced by the composite mapping $G \circ T$ in the iterative scheme of Theorem 16 .

(iv) The argument techniques of Theorem 16 are very different from Cai and Bu's ones of [9, Lemma 2.5]. Because the composite mapping $G \circ T$ appears in the iterative scheme of Theorem 16, the proof of Theorem 16 depends on the argument techniques in [18], the inequality in 2uniformly smooth Banach spaces (see Lemma 1), the inequality in smooth and uniform convex Banach spaces (see Proposition 2), and the properties of the strongly positive linear bounded operator (see Lemmas 15), the Banach limit (see Lemma 5), and the strongly accretive and strictly pseudocontractive mapping (see Lemma 7).

\section{GSVI with Hierarchical Fixed Point Problem Constraint for a Countable Family of Nonexpansive mappings}

In this section, we propose our hybrid explicit viscosity scheme for solving the GSVI (13) with hierarchical fixed point problem constraint for a countable family of nonexpansive mappings and show the strong convergence theorem.
Theorem 19. Let $C$ be a nonempty closed convex subset of a uniformly convex and 2-uniformly smooth Banach space $X$ such that $C \pm C \subset C$. Let $\Pi_{C}$ be a sunny nonexpansive retraction from $X$ onto $C$. Let the mapping $B_{i}: C \rightarrow X$ be $\alpha_{i}$-inverse-strongly accretive for $i=1,2$. Let $\left\{S_{n}\right\}_{n=0}^{\infty}$ be an infinite family of nonexpansive mappings of $C$ into itself such that $\Delta=\bigcap_{i=0}^{\infty} \operatorname{Fix}\left(S_{i}\right) \cap \Omega \neq \emptyset$, where $\Omega$ is the fixed point set of the mapping $G=\Pi_{C}\left(I-\mu_{1} B_{1}\right) \Pi_{C}\left(I-\mu_{2} B_{2}\right)$ with $0<\mu_{i}<\alpha_{i} / \kappa^{2}$ for $i=1,2$. Let $f: C \rightarrow C$ be a fixed contractive map with coefficient $\beta \in(0,1)$, let $F: C \rightarrow C$ be $\alpha$ strongly accretive and $\lambda$-strictly pseudocontractive with $\alpha+\lambda>$ 1 , and let $A: C \rightarrow C$ be a $\bar{\gamma}$-strongly positive linear bounded operator with $0<\bar{\gamma}-\beta \leq 1$. Given sequences $\left\{\lambda_{n}\right\}_{n=0}^{\infty},\left\{\mu_{n}\right\}_{n=0}^{\infty}$ in $[0,1]$ and $\left\{\alpha_{n}\right\}_{n=0}^{\infty},\left\{\beta_{n}\right\}_{n=0}^{\infty}$ in $(0,1]$, suppose that there hold the following conditions:

(i) $\lim _{n \rightarrow \infty} \beta_{n}=0$ and $\sum_{n=0}^{\infty} \beta_{n}=\infty$;

(ii) $\lim _{n \rightarrow \infty}\left(\lambda_{n} \mu_{n}\right) / \beta_{n}=0$;

(iii) $\left\{\alpha_{n}\right\} \subset[a, b]$ for some $a, b \in(0,1)$;

(iv) $\sum_{n=0}^{\infty}\left(\left|\alpha_{n+1}-\alpha_{n}\right|+\left|\beta_{n+1}-\beta_{n}\right|+\left|\lambda_{n+1}-\lambda_{n}\right|+\left|\mu_{n+1}-\mu_{n}\right|\right)<$ $\infty$.

Assume that $\sum_{n=0}^{\infty} \sup _{x \in D}\left\|S_{n+1} x-S_{n} x\right\|<\infty$ for any bounded subset $D$ of $C$ and let $S$ be a mapping of $C$ into itself defined by $S x=\lim _{n \rightarrow \infty} S_{n} x$ for all $x \in C$ and suppose that $\operatorname{Fix}(S)=$ $\bigcap_{n=0}^{\infty} \operatorname{Fix}\left(S_{n}\right)$. Then, for any given point $x_{0} \in C$, the sequence $\left\{x_{n}\right\}$ generated by

$$
\begin{aligned}
y_{n}= & \alpha_{n} x_{n}+\left(1-\alpha_{n}\right) G\left(S_{n} x_{n}\right), \\
x_{n+1}= & \beta_{n} f\left(x_{n}\right)+\left(I-\beta_{n} A\right) \\
& \times\left[G\left(S_{n} y_{n}\right)-\lambda_{n} \mu_{n} F G\left(S_{n} y_{n}\right)\right], \\
& \forall n \geq 0,
\end{aligned}
$$

converges strongly to $p \in \Delta$, which is the unique solution in $\Delta$ to the VIP:

$$
\langle(A-f) p, J(p-u)\rangle \leq 0, \quad \forall u \in \Delta
$$

Proof. First, let us show that $\left\{x_{n}\right\}$ is bounded. Indeed, taking a fixed $u \in \Delta$ arbitrarily, we have

$$
\begin{aligned}
\left\|y_{n}-u\right\| & =\left\|\alpha_{n} x_{n}+\left(1-\alpha_{n}\right) G\left(S_{n} x_{n}\right)-u\right\| \\
& \leq \alpha_{n}\left\|x_{n}-u\right\|+\left(1-\alpha_{n}\right)\left\|G\left(S_{n} x_{n}\right)-u\right\| \\
& \leq \alpha_{n}\left\|x_{n}-u\right\|+\left(1-\alpha_{n}\right)\left\|S_{n} x_{n}-u\right\| \\
& \leq \alpha_{n}\left\|x_{n}-u\right\|+\left(1-\alpha_{n}\right)\left\|x_{n}-u\right\|=\left\|x_{n}-u\right\| .
\end{aligned}
$$

So, $\left\|y_{n}-u\right\| \leq\left\|x_{n}-u\right\|$ for all $n \geq 0$. Taking into account $\lim _{n \rightarrow \infty}\left(\lambda_{n} \mu_{n}\right) / \beta_{n}=0$, we may assume, without loss of 
generality, that $\lambda_{n} \mu_{n} \leq \beta_{n} \leq\|A\|^{-1}$ for all $n \geq 0$. Thus, by Lemma 7 (ii), we have

$$
\begin{aligned}
\| x_{n+1} & -u \| \\
= & \| \beta_{n}\left(f\left(x_{n}\right)-f(u)\right)+\left(I-\beta_{n} A\right) \\
& \times\left[G\left(S_{n} y_{n}\right)-\lambda_{n} \mu_{n} F G\left(S_{n} y_{n}\right)-u\right]-\beta_{n}(A-f) u \| \\
\leq & \beta_{n}\left\|f\left(x_{n}\right)-f(u)\right\| \\
& +\left\|\left(I-\beta_{n} A\right)\left[G\left(S_{n} y_{n}\right)-\lambda_{n} \mu_{n} F G\left(S_{n} y_{n}\right)-u\right]\right\| \\
& +\beta_{n}\|(A-f) u\| \\
\leq & \beta_{n} \beta\left\|x_{n}-u\right\|+\left(1-\beta_{n} \bar{\gamma}\right) \\
& \times\left[\left\|\left(I-\lambda_{n} \mu_{n} F\right) G\left(S_{n} y_{n}\right)-\left(I-\lambda_{n} \mu_{n} F\right) G\left(S_{n} u\right)\right\|\right. \\
& \left.+\left\|\left(I-\lambda_{n} \mu_{n} F\right) G\left(S_{n} u\right)-u\right\|\right]+\beta_{n}\|(A-f) u\| \\
\leq & \beta_{n} \beta\left\|x_{n}-u\right\|+\left(1-\beta_{n} \bar{\gamma}\right) \\
& \times\left(1-\lambda_{n} \mu_{n}\left(1-\sqrt{\frac{1-\alpha}{\lambda}}\right)\right)\left\|G\left(S_{n} y_{n}\right)-G\left(S_{n} u\right)\right\| \\
& +\left(1-\beta_{n} \bar{\gamma}\right) \lambda_{n} \mu_{n}\|F u\|+\beta_{n}\|(A-f) u\| \\
\leq & \beta_{n} \beta\left\|x_{n}-u\right\|+\left(1-\beta_{n} \bar{\gamma}\right)\left(1-\lambda_{n} \mu_{n}\left(1-\sqrt{\frac{1-\alpha}{\lambda}}\right)\right) \\
& \times\left\|S_{n} y_{n}-S_{n} u\right\|+\lambda_{n} \mu_{n}\|F u\|+\beta_{n}\|(A-f) u\| \\
\leq & \beta_{n} \beta\left\|x_{n}-u\right\|+\left(1-\beta_{n} \bar{\gamma}\right)\left\|y_{n}-u\right\| \\
& +\lambda_{n} \mu_{n}\|F u\|+\beta_{n}\|(A-f) u\| \\
\leq & \beta_{n} \beta\left\|x_{n}-u\right\|+\left(1-\beta_{n} \bar{\gamma}\right)\left\|x_{n}-u\right\| \\
& +\beta_{n}\|F u\|+\beta_{n}\|(A-f) u\| \\
= & \left(1-\beta_{n}(\bar{\gamma}-\beta)\right)\left\|x_{n}-u\right\| \\
& \beta_{n}(\bar{\gamma}-\beta) \frac{\|(A-f) u\|+\|F u\|}{\bar{\gamma}-\beta} \\
& (A-f) u\|+\| F u \| \\
& \\
&
\end{aligned}
$$

By induction,

$$
\left\|x_{n}-u\right\| \leq \max \left\{\left\|x_{0}-u\right\|, \frac{\|(A-f) u\|+\|F u\|}{\bar{\gamma}-\beta}\right\}, \quad \forall n \geq 0 .
$$

Thus, $\left\{x_{n}\right\}$ is bounded and so is $\left\{y_{n}\right\}$. Because $G$ and $S_{n}$ are nonexpansive for all $n \geq 0, f$ is contractive, and $F$ is Lipschitzian, $\left\{S_{n} x_{n}\right\},\left\{S_{n} y_{n}\right\},\left\{G\left(S_{n} x_{n}\right)\right\},\left\{G\left(S_{n} y_{n}\right)\right\},\left\{f\left(x_{n}\right)\right\}$, and $\left\{F G\left(S_{n} y_{n}\right)\right\}$ are bounded. From conditions (i) and (ii) we have

$$
\begin{aligned}
\| x_{n+1}- & G\left(S_{n} y_{n}\right) \| \\
=\beta_{n} & \|\left(f\left(x_{n}\right)-A G\left(S_{n} y_{n}\right)\right)+\left(I-\beta_{n} A\right) \\
& \times\left(G\left(S_{n} y_{n}\right)-\lambda_{n} \mu_{n} F G\left(S_{n} y_{n}\right)-G\left(S_{n} y_{n}\right)\right) \| \\
\leq & \beta_{n}\left\|f\left(x_{n}\right)-A G\left(S_{n} y_{n}\right)\right\| \\
& +\left(1-\beta_{n} \bar{\gamma}\right) \lambda_{n} \mu_{n}\left\|F G\left(S_{n} y_{n}\right)\right\| \\
\leq & \beta_{n}\left\|f\left(x_{n}\right)-A G\left(S_{n} y_{n}\right)\right\| \\
& +\lambda_{n} \mu_{n}\left\|F G\left(S_{n} y_{n}\right)\right\| \longrightarrow 02 \text { as } n \longrightarrow \infty .
\end{aligned}
$$

Now, we claim that

$$
\left\|x_{n+1}-x_{n}\right\| \longrightarrow 0 \quad \text { as } n \longrightarrow \infty \text {. }
$$

In order to prove (90), we estimate $\left\|x_{n+1}-x_{n}\right\|$ first. From (84), we have

$$
\begin{gathered}
y_{n}=\alpha_{n} x_{n}+\left(1-\alpha_{n}\right) G\left(S_{n} x_{n}\right), \\
y_{n-1}=\alpha_{n-1} x_{n-1}+\left(1-\alpha_{n-1}\right) G\left(S_{n-1} x_{n-1}\right) .
\end{gathered}
$$

Simple calculations show that

$$
\begin{aligned}
y_{n}-y_{n-1}=(1 & \left.-\alpha_{n}\right)\left(G\left(S_{n} x_{n}\right)-G\left(S_{n-1} x_{n-1}\right)\right) \\
& +\alpha_{n}\left(x_{n}-x_{n-1}\right)+\left(x_{n-1}-G\left(S_{n-1} x_{n-1}\right)\right) \\
& \times\left(\alpha_{n}-\alpha_{n-1}\right) .
\end{aligned}
$$

It follows that

$$
\begin{aligned}
\| y_{n}- & y_{n-1} \| \\
\leq & \left(1-\alpha_{n}\right)\left\|G\left(S_{n} x_{n}\right)-G\left(S_{n-1} x_{n-1}\right)\right\|+\alpha_{n}\left\|x_{n}-x_{n-1}\right\| \\
& +\left\|x_{n-1}-G\left(S_{n-1} x_{n-1}\right)\right\|\left|\alpha_{n}-\alpha_{n-1}\right| \\
\leq & \left(1-\alpha_{n}\right)\left\|S_{n} x_{n}-S_{n-1} x_{n-1}\right\|+\alpha_{n}\left\|x_{n}-x_{n-1}\right\| \\
& +\left\|x_{n-1}-G\left(S_{n-1} x_{n-1}\right)\right\|\left|\alpha_{n}-\alpha_{n-1}\right| \\
\leq & \left(1-\alpha_{n}\right)\left(\left\|S_{n} x_{n}-S_{n} x_{n-1}\right\|+\left\|S_{n} x_{n-1}-S_{n-1} x_{n-1}\right\|\right) \\
& +\alpha_{n}\left\|x_{n}-x_{n-1}\right\|+\left\|x_{n-1}-G\left(S_{n-1} x_{n-1}\right)\right\|\left|\alpha_{n}-\alpha_{n-1}\right| \\
\leq & \left(1-\alpha_{n}\right)\left(\left\|x_{n}-x_{n-1}\right\|+\left\|S_{n} x_{n-1}-S_{n-1} x_{n-1}\right\|\right) \\
& +\alpha_{n}\left\|x_{n}-x_{n-1}\right\|+\left\|x_{n-1}-G\left(S_{n-1} x_{n-1}\right)\right\|\left|\alpha_{n}-\alpha_{n-1}\right| \\
\leq & \left\|x_{n}-x_{n-1}\right\|+\left\|x_{n-1}-G\left(S_{n-1} x_{n-1}\right)\right\|\left|\alpha_{n}-\alpha_{n-1}\right| \\
& +\left\|S_{n} x_{n-1}-S_{n-1} x_{n-1}\right\| .
\end{aligned}
$$


In the meantime, it follows from (84) that

$$
\begin{aligned}
x_{n+1}= & \beta_{n} f\left(x_{n}\right)+\left(I-\beta_{n} A\right)\left[G\left(S_{n} y_{n}\right)-\lambda_{n} \mu_{n} F G\left(S_{n} y_{n}\right)\right], \\
x_{n}= & \beta_{n-1} f\left(x_{n-1}\right)+\left(I-\beta_{n-1} A\right) \\
& \times\left[G\left(S_{n-1} y_{n-1}\right)-\lambda_{n-1} \mu_{n-1} F G\left(S_{n-1} y_{n-1}\right)\right] .
\end{aligned}
$$

Simple calculations show that

$$
\begin{aligned}
x_{n+1} & -x_{n} \\
= & \left(\beta_{n}-\beta_{n-1}\right) f\left(x_{n-1}\right)+\beta_{n}\left(f\left(x_{n}\right)-f\left(x_{n-1}\right)\right) \\
& +\left(\beta_{n-1}-\beta_{n}\right) A\left(I-\lambda_{n-1} \mu_{n-1} F\right) G\left(S_{n-1} y_{n-1}\right) \\
& +\left(I-\beta_{n} A\right)\left[\left(I-\lambda_{n} \mu_{n} F\right) G\left(S_{n} y_{n}\right)\right. \\
& \left.-\left(I-\lambda_{n-1} \mu_{n-1} F\right) G\left(S_{n-1} y_{n-1}\right)\right] \\
= & \left(\beta_{n}-\beta_{n-1}\right) f\left(x_{n-1}\right)+\beta_{n}\left(f\left(x_{n}\right)-f\left(x_{n-1}\right)\right) \\
& +\left(\beta_{n-1}-\beta_{n}\right) A\left(I-\lambda_{n-1} \mu_{n-1} F\right) G\left(S_{n-1} y_{n-1}\right) \\
& +\left(I-\beta_{n} A\right)\left[\left(I-\lambda_{n} \mu_{n} F\right) G\left(S_{n} y_{n}\right)\right. \\
& -\left(I-\lambda_{n} \mu_{n} F\right) G\left(S_{n-1} y_{n-1}\right) \\
& \left.+\left(\lambda_{n-1} \mu_{n-1}-\lambda_{n} \mu_{n}\right) F G\left(S_{n-1} y_{n-1}\right)\right] .
\end{aligned}
$$

It follows from Lemma 7 (ii) and (93) that

$$
\begin{aligned}
& \left\|x_{n+1}-x_{n}\right\| \\
& \leq\left|\beta_{n}-\beta_{n-1}\right|\left\|f\left(x_{n-1}\right)\right\|+\beta_{n}\left\|f\left(x_{n}\right)-f\left(x_{n-1}\right)\right\| \\
& +\left|\beta_{n-1}-\beta_{n}\right|\left\|A\left(I-\lambda_{n-1} \mu_{n-1} F\right) G\left(S_{n-1} y_{n-1}\right)\right\| \\
& +\left(1-\beta_{n} \bar{\gamma}\right)\left[\|\left(I-\lambda_{n} \mu_{n} F\right) G\left(S_{n} y_{n}\right)\right. \\
& -\left(I-\lambda_{n} \mu_{n} F\right) G\left(S_{n-1} y_{n-1}\right) \| \\
& \left.+\left|\lambda_{n-1} \mu_{n-1}-\lambda_{n} \mu_{n}\right|\left\|F G\left(S_{n-1} y_{n-1}\right)\right\|\right] \\
& \leq\left|\beta_{n}-\beta_{n-1}\right|\left\|f\left(x_{n-1}\right)\right\|+\beta_{n} \beta\left\|x_{n}-x_{n-1}\right\| \\
& +\left|\beta_{n-1}-\beta_{n}\right|\left\|A\left(I-\lambda_{n-1} \mu_{n-1} F\right) G\left(S_{n-1} y_{n-1}\right)\right\| \\
& +\left(1-\beta_{n} \bar{\gamma}\right)\left[\left(1-\lambda_{n} \mu_{n}\left(1-\sqrt{\frac{1-\alpha}{\lambda}}\right)\right)\right. \\
& \quad\left[\left\|G\left(S_{n} y_{n}\right)-G\left(S_{n-1} y_{n-1}\right)\right\|\right. \\
& \left.+\left|\beta_{n-1} \mu_{n}-\lambda_{n-1} \mu_{n-1}\right|\left\|F G\left(S_{n-1} y_{n-1}\right)\right\|\right] \\
& +\left|\beta_{n}-\beta_{n-1}\right|\left\|f\left(x_{n-1}\right)\right\|+\beta_{n} \beta\left\|x_{n}-x_{n-1}\right\| \\
& \left.+\mid \beta_{n-1} \mu_{n-1} F\right) G\left(S_{n-1} y_{n-1}\right) \|
\end{aligned}
$$

$+\left(1-\beta_{n} \bar{\gamma}\right)\left[\left\|S_{n} y_{n}-S_{n-1} y_{n-1}\right\|\right.$

$$
\left.+\left|\lambda_{n} \mu_{n}-\lambda_{n-1} \mu_{n-1}\right|\left\|F G\left(S_{n-1} y_{n-1}\right)\right\|\right]
$$

$\leq\left|\beta_{n}-\beta_{n-1}\right|\left\|f\left(x_{n-1}\right)\right\|+\beta_{n} \beta\left\|x_{n}-x_{n-1}\right\|$

$+\left|\beta_{n-1}-\beta_{n}\right|\left\|A\left(I-\lambda_{n-1} \mu_{n-1} F\right) G\left(S_{n-1} y_{n-1}\right)\right\|$

$+\left(1-\beta_{n} \bar{\gamma}\right)\left[\left\|S_{n} y_{n}-S_{n} y_{n-1}\right\|+\left\|S_{n} y_{n-1}-S_{n-1} y_{n-1}\right\|\right.$

$\left.+\left|\lambda_{n} \mu_{n}-\lambda_{n-1} \mu_{n-1}\right|\left\|F G\left(S_{n-1} y_{n-1}\right)\right\|\right]$

$\leq\left|\beta_{n}-\beta_{n-1}\right|\left\|f\left(x_{n-1}\right)\right\|+\beta_{n} \beta\left\|x_{n}-x_{n-1}\right\|$

$+\left|\beta_{n-1}-\beta_{n}\right|\left\|A\left(I-\lambda_{n-1} \mu_{n-1} F\right) G\left(S_{n-1} y_{n-1}\right)\right\|$

$+\left(1-\beta_{n} \bar{\gamma}\right)\left[\left\|y_{n}-y_{n-1}\right\|+\left\|S_{n} y_{n-1}-S_{n-1} y_{n-1}\right\|\right.$

$\left.+\left|\lambda_{n} \mu_{n}-\lambda_{n-1} \mu_{n-1}\right|\left\|F G\left(S_{n-1} y_{n-1}\right)\right\|\right]$

$\leq\left|\beta_{n}-\beta_{n-1}\right|\left\|f\left(x_{n-1}\right)\right\|+\beta_{n} \beta\left\|x_{n}-x_{n-1}\right\|$

$+\left|\beta_{n-1}-\beta_{n}\right|\left\|A\left(I-\lambda_{n-1} \mu_{n-1} F\right) G\left(S_{n-1} y_{n-1}\right)\right\|$

$+\left(1-\beta_{n} \bar{\gamma}\right)\left[\left\|x_{n}-x_{n-1}\right\|+\left\|x_{n-1}-G\left(S_{n-1} x_{n-1}\right)\right\|\right.$

$\times\left|\alpha_{n}-\alpha_{n-1}\right|+\left\|S_{n} x_{n-1}-S_{n-1} x_{n-1}\right\|$

$+\left\|S_{n} y_{n-1}-S_{n-1} y_{n-1}\right\|$

$\left.+\left|\lambda_{n} \mu_{n}-\lambda_{n-1} \mu_{n-1}\right|\left\|F G\left(S_{n-1} y_{n-1}\right)\right\|\right]$

$\leq\left(1-\beta_{n}(\bar{\gamma}-\beta)\right)\left\|x_{n}-x_{n-1}\right\|+\left|\beta_{n}-\beta_{n-1}\right|$

$\times\left(\left\|f\left(x_{n-1}\right)\right\|+\left\|A\left(I-\lambda_{n-1} \mu_{n-1} F\right) G\left(S_{n-1} y_{n-1}\right)\right\|\right)$

$+\left\|x_{n-1}-G\left(S_{n-1} x_{n-1}\right)\right\|\left|\alpha_{n}-\alpha_{n-1}\right|+\left|\lambda_{n} \mu_{n}-\lambda_{n-1} \mu_{n-1}\right|$

$\times\left\|F G\left(S_{n-1} y_{n-1}\right)\right\|+\left\|S_{n} x_{n-1}-S_{n-1} x_{n-1}\right\|$

$+\left\|S_{n} y_{n-1}-S_{n-1} y_{n-1}\right\|$

$\leq\left(1-\beta_{n}(\bar{\gamma}-\beta)\right)\left\|x_{n}-x_{n-1}\right\|+\left|\beta_{n}-\beta_{n-1}\right| M_{0}$

$+M_{0}\left|\alpha_{n}-\alpha_{n-1}\right|$

$+\left|\lambda_{n} \mu_{n}-\lambda_{n-1} \mu_{n-1}\right| M_{0}+\left\|S_{n} x_{n-1}-S_{n-1} x_{n-1}\right\|$

$+\left\|S_{n} y_{n-1}-S_{n-1} y_{n-1}\right\|$

$=\left(1-\beta_{n}(\bar{\gamma}-\beta)\right)\left\|x_{n}-x_{n-1}\right\|$

$+M_{0}\left(\left|\alpha_{n}-\alpha_{n-1}\right|+\left|\beta_{n}-\beta_{n-1}\right|+\left|\lambda_{n} \mu_{n}-\lambda_{n-1} \mu_{n-1}\right|\right)$

$+\left\|S_{n} x_{n-1}-S_{n-1} x_{n-1}\right\|+\left\|S_{n} y_{n-1}-S_{n-1} y_{n-1}\right\|$,

$\leq\left(1-\beta_{n}(\bar{\gamma}-\beta)\right)\left\|x_{n}-x_{n-1}\right\|$

$+M_{0}\left(\left|\alpha_{n}-\alpha_{n-1}\right|+\left|\beta_{n}-\beta_{n-1}\right|+\left|\lambda_{n}-\lambda_{n-1}\right|\right.$

$\left.+\left|\mu_{n}-\mu_{n-1}\right|\right)+\left\|S_{n} x_{n-1}-S_{n-1} x_{n-1}\right\|$

$+\left\|S_{n} y_{n-1}-S_{n-1} y_{n-1}\right\|$, 
where $\sup _{n \geq 0}\left\{\left\|f\left(x_{n}\right)\right\|+\left\|A\left(I-\lambda_{n} \mu_{n} F\right) G\left(S_{n} y_{n}\right)\right\|+\left\|F G\left(S_{n} y_{n}\right)\right\|+\right.$ $\left.\left\|x_{n}-G\left(S_{n} x_{n}\right)\right\|\right\} \leq M_{0}$ for some $M_{0}>0$. Since it follows from conditions (i) and (iv) that $\sum_{n=0}^{\infty} \beta_{n}(\bar{\gamma}-\beta)=\infty$ and

$$
\begin{aligned}
& \sum_{n=0}^{\infty} M_{0}\left(\left|\alpha_{n}-\alpha_{n-1}\right|+\left|\beta_{n}-\beta_{n-1}\right|\right. \\
& \left.+\left|\lambda_{n}-\lambda_{n-1}\right|+\left|\mu_{n}-\mu_{n-1}\right|\right)<\infty,
\end{aligned}
$$

applying Lemma 3 to (96), we obtain from the assumption on $\left\{S_{n}\right\}$ that

$$
\lim _{n \rightarrow \infty}\left\|x_{n+1}-x_{n}\right\|=0 .
$$

By condition (iii) and (84), we have

$$
\begin{aligned}
\left\|y_{n}-x_{n}\right\|=\left(1-\alpha_{n}\right)\left\|G\left(S_{n} x_{n}\right)-x_{n}\right\| \\
\leq(1-a)\left(\left\|G\left(S_{n} x_{n}\right)-G\left(S_{n} y_{n}\right)\right\|\right. \\
\left.+\left\|G\left(S_{n} y_{n}\right)-x_{n+1}\right\|+\left\|x_{n+1}-x_{n}\right\|\right) \\
\leq(1-a)\left(\left\|x_{n}-y_{n}\right\|+\left\|G\left(S_{n} y_{n}\right)-x_{n+1}\right\|\right. \\
\left.+\left\|x_{n+1}-x_{n}\right\|\right),
\end{aligned}
$$

which implies that

$$
\left\|y_{n}-x_{n}\right\| \leq \frac{1-a}{a}\left(\left\|G\left(S_{n} y_{n}\right)-x_{n+1}\right\|+\left\|x_{n+1}-x_{n}\right\|\right) .
$$

This together with (89)-(90) implies that

$$
\lim _{n \rightarrow \infty}\left\|x_{n}-y_{n}\right\|=0 \text {. }
$$

So, we obtain

$$
\begin{aligned}
\left\|x_{n}-G\left(S_{n} x_{n}\right)\right\| & \leq\left\|x_{n}-y_{n}\right\|+\left\|y_{n}-G\left(S_{n} x_{n}\right)\right\| \\
& \leq\left\|x_{n}-y_{n}\right\|+\alpha_{n}\left\|x_{n}-G\left(S_{n} x_{n}\right)\right\| \\
& \leq\left\|x_{n}-y_{n}\right\|+b\left\|x_{n}-G\left(S_{n} x_{n}\right)\right\|,
\end{aligned}
$$

which implies that

$$
\left\|x_{n}-G\left(S_{n} x_{n}\right)\right\| \leq \frac{1}{1-b}\left\|x_{n}-y_{n}\right\|,
$$

and hence

$$
\lim _{n \rightarrow \infty}\left\|x_{n}-G\left(S_{n} x_{n}\right)\right\|=0 .
$$

Let $u \in \Delta$. Now, we show that $\lim _{n \rightarrow \infty}\left\|x_{n}-S x_{n}\right\|=0$ and $\lim _{n \rightarrow \infty}\left\|x_{n}-G x_{n}\right\|=0$.

Indeed, for simplicity, put $v=\Pi_{C}\left(u-\mu_{2} B_{2} u\right), \widehat{x}_{n}=S_{n} x_{n}$, $u_{n}=\Pi_{C}\left(\widehat{x}_{n}-\mu_{2} B_{2} \widehat{x}_{n}\right)$, and $v_{n}=\Pi_{C}\left(u_{n}-\mu_{1} B_{1} u_{n}\right)$. Then, $u=\Pi_{C}\left(v-\mu_{1} B_{1} v\right)$ and $v_{n}=G \widehat{x}_{n}=G\left(S_{n} x_{n}\right)$ for all $n \geq 0$. It is clear from (84) that

$$
\begin{aligned}
\left\|y_{n}-u\right\|^{2} & \leq \alpha_{n}\left\|x_{n}-u\right\|^{2}+\left(1-\alpha_{n}\right)\left\|G\left(S_{n} x_{n}\right)-u\right\|^{2} \\
& =\alpha_{n}\left\|x_{n}-u\right\|^{2}+\left(1-\alpha_{n}\right)\left\|v_{n}-u\right\|^{2} .
\end{aligned}
$$

Utilizing Lemma 12, we have

$$
\begin{aligned}
\left\|u_{n}-v\right\|^{2} & =\left\|\Pi_{C}\left(\widehat{x}_{n}-\mu_{2} B_{2} \widehat{x}_{n}\right)-\Pi_{C}\left(u-\mu_{2} B_{2} u\right)\right\|^{2} \\
& \leq\left\|\widehat{x}_{n}-u-\mu_{2}\left(B_{2} \widehat{x}_{n}-B_{2} u\right)\right\|^{2} \\
& \leq\left\|\widehat{x}_{n}-u\right\|^{2}-2 \mu_{2}\left(\alpha_{2}-\kappa^{2} \mu_{2}\right)\left\|B_{2} \widehat{x}_{n}-B_{2} u\right\|^{2},
\end{aligned}
$$

$$
\begin{aligned}
\left\|v_{n}-u\right\|^{2} & =\left\|\Pi_{C}\left(u_{n}-\mu_{1} B_{1} u_{n}\right)-\Pi_{C}\left(v-\mu_{1} B_{1} v\right)\right\|^{2} \\
& \leq\left\|u_{n}-v-\mu_{1}\left(B_{1} u_{n}-B_{1} v\right)\right\|^{2} \\
& \leq\left\|u_{n}-v\right\|^{2}-2 \mu_{1}\left(\alpha_{1}-\kappa^{2} \mu_{1}\right)\left\|B_{1} u_{n}-B_{1} v\right\|^{2} .
\end{aligned}
$$

Substituting (106) for (107), we obtain

$$
\begin{aligned}
\left\|v_{n}-u\right\|^{2} \leq & \left\|\widehat{x}_{n}-u\right\|^{2}-2 \mu_{2}\left(\alpha_{2}-\kappa^{2} \mu_{2}\right) \\
& \times\left\|B_{2} \widehat{x}_{n}-B_{2} u\right\|^{2}-2 \mu_{1}\left(\alpha_{1}-\kappa^{2} \mu_{1}\right) \\
& \times\left\|B_{1} u_{n}-B_{1} v\right\|^{2} \\
\leq & \left\|x_{n}-u\right\|^{2}-2 \mu_{2}\left(\alpha_{2}-\kappa^{2} \mu_{2}\right) \\
& \times\left\|B_{2} \widehat{x}_{n}-B_{2} u\right\|^{2}-2 \mu_{1}\left(\alpha_{1}-\kappa^{2} \mu_{1}\right) \\
& \times\left\|B_{1} u_{n}-B_{1} v\right\|^{2},
\end{aligned}
$$

which together with (105) implies that

$$
\begin{gathered}
\left\|y_{n}-u\right\|^{2} \leq \alpha_{n}\left\|x_{n}-u\right\|^{2}+\left(1-\alpha_{n}\right)\left\|v_{n}-u\right\|^{2} \\
\leq \alpha_{n}\left\|x_{n}-u\right\|^{2}+\left(1-\alpha_{n}\right) \\
\times\left[\left\|x_{n}-u\right\|^{2}-2 \mu_{2}\left(\alpha_{2}-\kappa^{2} \mu_{2}\right)\right. \\
\times\left\|B_{2} \widehat{x}_{n}-B_{2} u\right\|^{2} \\
\left.\quad-2 \mu_{1}\left(\alpha_{1}-\kappa^{2} \mu_{1}\right)\left\|B_{1} u_{n}-B_{1} v\right\|^{2}\right] \\
=\left\|x_{n}-u\right\|^{2}-2\left(1-\alpha_{n}\right) \\
\times\left[\mu_{2}\left(\alpha_{2}-\kappa^{2} \mu_{2}\right)\left\|B_{2} \widehat{x}_{n}-B_{2} u\right\|^{2}\right. \\
\left.\quad+\mu_{1}\left(\alpha_{1}-\kappa^{2} \mu_{1}\right)\left\|B_{1} u_{n}-B_{1} v\right\|^{2}\right] .
\end{gathered}
$$

It immediately follows that

$$
\begin{aligned}
& 2\left(1-\alpha_{n}\right)\left[\mu_{2}\left(\alpha_{2}-\kappa^{2} \mu_{2}\right)\left\|B_{2} \widehat{x}_{n}-B_{2} u\right\|^{2}\right. \\
& \left.+\mu_{1}\left(\alpha_{1}-\kappa^{2} \mu_{1}\right)\left\|B_{1} u_{n}-B_{1} v\right\|^{2}\right] \\
& \leq\left\|x_{n}-u\right\|^{2}-\left\|y_{n}-u\right\|^{2} \\
& \leq\left(\left\|x_{n}-u\right\|+\left\|y_{n}-u\right\|\right)\left\|x_{n}-y_{n}\right\| .
\end{aligned}
$$


Since $\left\{x_{n}\right\}$ and $\left\{y_{n}\right\}$ are bounded and $0<\mu_{i}<\alpha_{i} / \kappa^{2}$ for $i=$ 1,2 , we deduce from (101) and condition (iii) that

$$
\lim _{n \rightarrow \infty}\left\|B_{2} \widehat{x}_{n}-B_{2} u\right\|=0, \quad \lim _{n \rightarrow \infty}\left\|B_{1} u_{n}-B_{1} v\right\|=0 .
$$

Utilizing Proposition 2 and Lemma 8, we have that there exists $g_{1}$ such that

$$
\begin{aligned}
\left\|u_{n}-v\right\|^{2}= & \left\|\Pi_{C}\left(\widehat{x}_{n}-\mu_{2} B_{2} \widehat{x}_{n}\right)-\Pi_{C}\left(u-\mu_{2} B_{2} u\right)\right\|^{2} \\
\leq & \left\langle\widehat{x}_{n}-\mu_{2} B_{2} \widehat{x}_{n}-\left(u-\mu_{2} B_{2} u\right), J\left(u_{n}-v\right)\right\rangle \\
= & \left\langle\widehat{x}_{n}-u, J\left(u_{n}-v\right)\right\rangle \\
& +\mu_{2}\left\langle B_{2} u-B_{2} \widehat{x}_{n}, J\left(u_{n}-v\right)\right\rangle \\
\leq & \frac{1}{2}\left[\left\|\widehat{x}_{n}-u\right\|^{2}+\left\|u_{n}-v\right\|^{2}\right. \\
& \left.\quad-g_{1}\left(\left\|\widehat{x}_{n}-u_{n}-(u-v)\right\|\right)\right] \\
& +\mu_{2}\left\|B_{2} u-B_{2} \widehat{x}_{n}\right\|\left\|u_{n}-v\right\|,
\end{aligned}
$$

which implies that

$$
\begin{aligned}
\left\|u_{n}-v\right\|^{2} \leq & \left\|\widehat{x}_{n}-u\right\|^{2}-g_{1}\left(\left\|\widehat{x}_{n}-u_{n}-(u-v)\right\|\right) \\
& +2 \mu_{2}\left\|B_{2} u-B_{2} \widehat{x}_{n}\right\|\left\|u_{n}-v\right\| .
\end{aligned}
$$

In the same way, we derive that there exists $g_{2}$ such that

$$
\begin{aligned}
\left\|v_{n}-u\right\|^{2} & =\left\|\Pi_{C}\left(u_{n}-\mu_{1} B_{1} u_{n}\right)-\Pi_{C}\left(v-\mu_{1} B_{1} v\right)\right\|^{2} \\
\leq & \left\langle u_{n}-\mu_{1} B_{1} u_{n}-\left(v-\mu_{1} B_{1} v\right), J\left(v_{n}-u\right)\right\rangle \\
& =\left\langle u_{n}-v, J\left(v_{n}-u\right)\right\rangle+\mu_{1}\left\langle B_{1} v-B_{1} u_{n}, J\left(v_{n}-u\right)\right\rangle \\
\leq & \frac{1}{2}\left[\left\|u_{n}-v\right\|^{2}+\left\|v_{n}-u\right\|^{2}\right. \\
& \left.\quad-g_{2}\left(\left\|u_{n}-v_{n}+(u-v)\right\|\right)\right] \\
& +\mu_{1}\left\|B_{1} v-B_{1} u_{n}\right\|\left\|v_{n}-u\right\|,
\end{aligned}
$$

which implies that

$$
\begin{aligned}
\left\|v_{n}-u\right\|^{2} \leq & \left\|u_{n}-v\right\|^{2}-g_{2}\left(\left\|u_{n}-v_{n}+(u-v)\right\|\right) \\
& +2 \mu_{1}\left\|B_{1} v-B_{1} u_{n}\right\|\left\|v_{n}-u\right\| .
\end{aligned}
$$

Substituting (113) for (115), we get

$$
\begin{aligned}
\| v_{n}- & u \|^{2} \\
\leq & \left\|\widehat{x}_{n}-u\right\|^{2}-g_{1}\left(\left\|\widehat{x}_{n}-u_{n}-(u-v)\right\|\right) \\
& -g_{2}\left(\left\|u_{n}-v_{n}+(u-v)\right\|\right) \\
& +2 \mu_{2}\left\|B_{2} u-B_{2} \widehat{x}_{n}\right\|\left\|u_{n}-v\right\| \\
& +2 \mu_{1}\left\|B_{1} v-B_{1} u_{n}\right\|\left\|v_{n}-u\right\| \\
\leq & \left\|x_{n}-u\right\|^{2}-g_{1}\left(\left\|\widehat{x}_{n}-u_{n}-(u-v)\right\|\right) \\
& -g_{2}\left(\left\|u_{n}-v_{n}+(u-v)\right\|\right) \\
& +2 \mu_{2}\left\|B_{2} u-B_{2} \widehat{x}_{n}\right\|\left\|u_{n}-v\right\| \\
& +2 \mu_{1}\left\|B_{1} v-B_{1} u_{n}\right\|\left\|v_{n}-u\right\|
\end{aligned}
$$

which together with (105) implies that

$$
\begin{aligned}
\left\|y_{n}-u\right\|^{2} \leq & \alpha_{n}\left\|x_{n}-u\right\|^{2}+\left(1-\alpha_{n}\right)\left\|v_{n}-u\right\|^{2} \\
\leq & \alpha_{n}\left\|x_{n}-u\right\|^{2}+\left(1-\alpha_{n}\right) \\
\times & {\left[\left\|x_{n}-u\right\|^{2}-g_{1}\left(\left\|\widehat{x}_{n}-u_{n}-(u-v)\right\|\right)\right.} \\
& -g_{2}\left(\left\|u_{n}-v_{n}+(u-v)\right\|\right) \\
& +2 \mu_{2}\left\|B_{2} u-B_{2} \widehat{x}_{n}\right\|\left\|u_{n}-v\right\| \\
& \left.+2 \mu_{1}\left\|B_{1} v-B_{1} u_{n}\right\|\left\|v_{n}-u\right\|\right] \\
= & x_{n}-u \|^{2}-\left(1-\alpha_{n}\right) \\
\times & {\left[g_{1}\left(\left\|\widehat{x}_{n}-u_{n}-(u-v)\right\|\right)\right.} \\
& \left.+g_{2}\left(\left\|u_{n}-v_{n}+(u-v)\right\|\right)\right]+2\left(1-\alpha_{n}\right) \\
\times & \left(\mu_{2}\left\|B_{2} u-B_{2} \widehat{x}_{n}\right\|\left\|u_{n}-v\right\|\right. \\
& \left.+\mu_{1}\left\|B_{1} v-B_{1} u_{n}\right\|\left\|v_{n}-u\right\|\right) .
\end{aligned}
$$

It immediately follows that

$$
\begin{gathered}
\left(1-\alpha_{n}\right)\left[g_{1}\left(\left\|\widehat{x}_{n}-u_{n}-(u-v)\right\|\right)\right. \\
\left.+g_{2}\left(\left\|u_{n}-v_{n}+(u-v)\right\|\right)\right] \\
\leq\left\|x_{n}-u\right\|^{2}-\left\|y_{n}-u\right\|^{2}+2\left(1-\alpha_{n}\right) \\
\times\left(\mu_{2}\left\|B_{2} u-B_{2} \widehat{x}_{n}\right\|\left\|u_{n}-v\right\|\right. \\
\left.+\mu_{1}\left\|B_{1} v-B_{1} u_{n}\right\|\left\|v_{n}-u\right\|\right) \\
\leq\left(\left\|x_{n}-u\right\|+\left\|y_{n}-u\right\|\right)\left\|x_{n}-y_{n}\right\| \\
+2 \mu_{2}\left\|B_{2} u-B_{2} \widehat{x}_{n}\right\|\left\|u_{n}-v\right\| \\
+2 \mu_{1}\left\|B_{1} v-B_{1} u_{n}\right\|\left\|v_{n}-u\right\| .
\end{gathered}
$$


Since $\left\{x_{n}\right\},\left\{y_{n}\right\},\left\{u_{n}\right\}$, and $\left\{v_{n}\right\}$ are bounded, we deduce from (101), (111), and condition (iii) that

$$
\begin{aligned}
& \lim _{n \rightarrow \infty} g_{1}\left(\left\|\widehat{x}_{n}-u_{n}-(u-v)\right\|\right)=0, \\
& \lim _{n \rightarrow \infty} g_{2}\left(\left\|u_{n}-v_{n}+(u-v)\right\|\right)=0 .
\end{aligned}
$$

Utilizing the properties of $g_{1}$ and $g_{2}$, we get

$$
\begin{aligned}
& \lim _{n \rightarrow \infty}\left\|\widehat{x}_{n}-u_{n}-(u-v)\right\|=0, \\
& \lim _{n \rightarrow \infty}\left\|u_{n}-v_{n}+(u-v)\right\|=0,
\end{aligned}
$$

which hence yields

$$
\begin{array}{r}
\left\|\widehat{x}_{n}-v_{n}\right\| \leq\left\|\widehat{x}_{n}-u_{n}-(u-v)\right\| \\
+\left\|u_{n}-v_{n}+(u-v)\right\| \longrightarrow 0 \\
\text { as } n \rightarrow \infty .
\end{array}
$$

That is,

$$
\lim _{n \rightarrow \infty}\left\|S_{n} x_{n}-G\left(S_{n} x_{n}\right)\right\|=\lim _{n \rightarrow \infty}\left\|\widehat{x}_{n}-v_{n}\right\|=0 .
$$

Note that

$$
\left\|x_{n}-S_{n} x_{n}\right\| \leq\left\|x_{n}-G\left(S_{n} x_{n}\right)\right\|+\left\|G\left(S_{n} x_{n}\right)-S_{n} x_{n}\right\| .
$$

So, from (104) and (122), we have

$$
\lim _{n \rightarrow \infty}\left\|x_{n}-S_{n} x_{n}\right\|=0,
$$

which together with (104) and the assumption on $\left\{S_{n}\right\}$ implies that

$$
\begin{aligned}
\left\|x_{n}-S x_{n}\right\| & \leq\left\|x_{n}-S_{n} x_{n}\right\|+\left\|S_{n} x_{n}-S x_{n}\right\| \\
\text { as } n \longrightarrow 0 & \longrightarrow \infty, \\
\left\|x_{n}-G x_{n}\right\| & \leq\left\|x_{n}-G\left(S_{n} x_{n}\right)\right\|+\left\|G\left(S_{n} x_{n}\right)-G x_{n}\right\| \\
& \leq\left\|x_{n}-G\left(S_{n} x_{n}\right)\right\|+\left\|S_{n} x_{n}-x_{n}\right\| \\
\text { as } n & \longrightarrow 0
\end{aligned}
$$

That is,

$$
\lim _{n \rightarrow \infty}\left\|x_{n}-S x_{n}\right\|=0, \quad \lim _{n \rightarrow \infty}\left\|x_{n}-G x_{n}\right\|=0 .
$$

Define a mapping

$$
W x=(1-\theta) S x+\theta G x, \quad \forall x \in C,
$$

where $\theta$ is a constant in $(0,1)$. Then, by Lemma 10 , we know that $\operatorname{Fix}(W)=\operatorname{Fix}(S) \cap \operatorname{Fix}(G)=\Delta$. We observe that

$$
\begin{aligned}
\left\|x_{n}-W x_{n}\right\| & =\left\|(1-\theta)\left(x_{n}-S x_{n}\right)+\theta\left(x_{n}-G x_{n}\right)\right\| \\
& \leq(1-\theta)\left\|x_{n}-S x_{n}\right\|+\left\|\theta x_{n}-G x_{n}\right\| .
\end{aligned}
$$

So, from (126), we get

$$
\lim _{n \rightarrow \infty}\left\|x_{n}-W x_{n}\right\|=0
$$

where $p$ is defined below. Now, we claim that

$$
\limsup _{n \rightarrow \infty}\left\langle(f-A) p, J\left(x_{n}-p\right)\right\rangle \leq 0 .
$$

Indeed, let $\left\{x_{t}\right\}$ be defined by

$$
x_{t}=t f\left(x_{t}\right)+(I-t A) W x_{t} .
$$

Then, as $t \rightarrow 0,\left\{x_{t}\right\}$ converges strongly to $p \in \operatorname{Fix}(W)=\Delta$, which by Proposition $\mathrm{CB}$ is the unique solution in $\Delta$ to the VIP:

$$
\langle(A-f) p, J(p-u)\rangle \leq 0, \quad \forall u \in \Delta .
$$

In terms of Lemma 6, we conclude from (129) that (130) holds. It is clear that

$$
\limsup _{n \rightarrow \infty}\left\langle(f-A) p, J\left(x_{n+1}-p\right)\right\rangle \leq 0 .
$$

Finally, let us show that $x_{n} \rightarrow p$ as $n \rightarrow \infty$. We observe that

$$
\begin{aligned}
\left\|y_{n}-p\right\|^{2} & \leq \alpha_{n}\left\|x_{n}-p\right\|^{2}+\left(1-\alpha_{n}\right)\left\|G\left(S_{n} x_{n}\right)-p\right\|^{2} \\
& \leq \alpha_{n}\left\|x_{n}-p\right\|^{2}+\left(1-\alpha_{n}\right)\left\|x_{n}-p\right\|^{2} \\
& =\left\|x_{n}-p\right\|^{2}
\end{aligned}
$$

and hence

$$
\begin{aligned}
\| x_{n+1}- & p \|^{2} \\
= & \| \beta_{n}\left(f\left(x_{n}\right)-f(p)\right)+\left(I-\beta_{n} A\right) \\
& \times\left[G\left(S_{n} y_{n}\right)-\lambda_{n} \mu_{n} F G\left(S_{n} y_{n}\right)-p\right]+\beta_{n}(f-A) p \|^{2} \\
\leq & \| \beta_{n}\left(f\left(x_{n}\right)-f(p)\right)+\left(I-\beta_{n} A\right) \\
& \times\left[G\left(S_{n} y_{n}\right)-\lambda_{n} \mu_{n} F G\left(S_{n} y_{n}\right)-p\right] \|^{2} \\
+ & 2 \beta_{n}\left\langle(f-A) p, J\left(x_{n+1}-p\right)\right\rangle \\
\leq & {\left[\beta_{n}\left\|f\left(x_{n}\right)-f(p)\right\|+\left(1-\beta_{n} \bar{\gamma}\right)\right.} \\
& \left.\times\left\|G\left(S_{n} y_{n}\right)-\lambda_{n} \mu_{n} F G\left(S_{n} y_{n}\right)-p\right\|\right]^{2} \\
+ & 2 \beta_{n}\left\langle(f-A) p, J\left(x_{n+1}-p\right)\right\rangle \\
= & {\left[\beta_{n}\left\|f\left(x_{n}\right)-f(p)\right\|+\left(1-\beta_{n} \bar{\gamma}\right)\right.} \\
& \times \|\left(I-\lambda_{n} \mu_{n} F\right) G\left(S_{n} y_{n}\right) \\
& \left.\quad-\left(I-\lambda_{n} \mu_{n} F\right) p-\lambda_{n} \mu_{n} F p \|\right]^{2} \\
+ & 2 \beta_{n}\left\langle(f-A) p, J\left(x_{n+1}-p\right)\right\rangle \\
& \times\left(\left\|\left(I-\beta_{n} \mu_{n} F\right) G x_{n}-p\right\|+\left(1-\beta_{n} \bar{\gamma}\right)\right. \\
& \quad\left(S_{n} y_{n}\right)-\left(I-\lambda_{n} \mu_{n} F\right) p \|
\end{aligned}
$$




$$
\begin{aligned}
& \leq\left[\beta_{n} \beta\left\|x_{n}-p\right\|+\left(1-\beta_{n} \bar{\gamma}\right)\right. \\
& \times\left(\left(1-\lambda_{n} \mu_{n}\left(1-\sqrt{\frac{1-\alpha}{\lambda}}\right)\right)\right. \\
& \left.\left.\times\left\|G\left(S_{n} y_{n}\right)-p\right\|+\lambda_{n} \mu_{n}\|F p\|\right)\right]^{2} \\
& +2 \beta_{n}\left\langle(f-A) p, J\left(x_{n+1}-p\right)\right\rangle \\
& \leq\left[\beta_{n} \beta\left\|x_{n}-p\right\|\right. \\
& \left.+\left(1-\beta_{n} \bar{\gamma}\right)\left(\left\|y_{n}-p\right\|+\lambda_{n} \mu_{n}\|F p\|\right)\right]^{2} \\
& +2 \beta_{n}\left\langle(f-A) p, J\left(x_{n+1}-p\right)\right\rangle \\
& \leq\left[\beta_{n} \beta\left\|x_{n}-p\right\|+\left(1-\beta_{n} \bar{\gamma}\right)\left\|x_{n}-p\right\|+\lambda_{n} \mu_{n}\|F p\|\right]^{2} \\
& +2 \beta_{n}\left\langle(f-A) p, J\left(x_{n+1}-p\right)\right\rangle \\
& =\left[\left(1-\beta_{n}(\bar{\gamma}-\beta)\right)\left\|x_{n}-p\right\|+\lambda_{n} \mu_{n}\|F p\|\right]^{2} \\
& +2 \beta_{n}\left\langle(f-A) p, J\left(x_{n+1}-p\right)\right\rangle \\
& =\left(1-\beta_{n}(\bar{\gamma}-\beta)\right)^{2}\left\|x_{n}-p\right\|^{2}+\lambda_{n} \mu_{n}\|F p\| \\
& \times\left[2\left(1-\beta_{n}(\bar{\gamma}-\beta)\right)\left\|x_{n}-p\right\|+\lambda_{n} \mu_{n}\|F p\|\right] \\
& +2 \beta_{n}\left\langle(f-A) p, J\left(x_{n+1}-p\right)\right\rangle \\
& \leq\left(1-\beta_{n}(\bar{\gamma}-\beta)\right)\left\|x_{n}-p\right\|^{2} \\
& +\lambda_{n} \mu_{n}\|F p\|\left(2\left\|x_{n}-p\right\|+\lambda_{n} \mu_{n}\|F p\|\right) \\
& +2 \beta_{n}\left\langle(f-A) p, J\left(x_{n+1}-p\right)\right\rangle \\
& =\left(1-\beta_{n}(\bar{\gamma}-\beta)\right)\left\|x_{n}-p\right\|^{2}+\beta_{n}(\bar{\gamma}-\beta) \cdot \frac{1}{\bar{\gamma}-\beta} \\
& \times\left[\frac{\lambda_{n} \mu_{n}}{\beta_{n}}\|F p\|\left(2\left\|x_{n}-p\right\|+\lambda_{n} \mu_{n}\|F p\|\right)\right. \\
& \left.+2\left\langle(f-A) p, J\left(x_{n+1}-p\right)\right\rangle\right] .
\end{aligned}
$$

Taking into account (133) and conditions (i) and (ii), we obtain that $\sum_{n=0}^{\infty}(\bar{\gamma}-\beta) \beta_{n}=\infty$ and

$$
\begin{array}{r}
\limsup _{n \rightarrow \infty} \frac{1}{\bar{\gamma}-\beta}\left[\frac{\lambda_{n} \mu_{n}}{\beta_{n}}\|F p\|\left(2\left\|x_{n}-p\right\|+\lambda_{n} \mu_{n}\|F p\|\right)\right. \\
\left.+2\left\langle(f-A) p, J\left(x_{n+1}-p\right)\right\rangle\right] \leq 0 .
\end{array}
$$

Therefore, applying Lemma 3 to (135), we infer that

$$
\lim _{n \rightarrow \infty}\left\|x_{n}-p\right\|=0
$$

This completes the proof.
Remark 20. It is worth pointing out that the sequences $\left\{\lambda_{n}\right\}$, $\left\{\mu_{n}\right\}$, and $\left\{\beta_{n}\right\}$ can be taken, which satisfy the conditions in Theorem 19. As a matter of fact, put $\lambda_{n}=(1+n)^{-5 / 6}, \mu_{n}=$ 1 , and $\beta_{n}=(1+n)^{-2 / 3}$ for all $n \geq 0$. Then, there hold the following statements:

(i) $\lim _{n \rightarrow \infty} \beta_{n}=0$ and $\sum_{n=0}^{\infty} \beta_{n}=\infty$;

(ii) $\lim _{n \rightarrow \infty}\left(\lambda_{n} \mu_{n}\right) / \beta_{n}=0$;

(iii) $\sum_{n=0}^{\infty}\left|\beta_{n+1}-\beta_{n}\right|<\infty, \sum_{n=0}^{\infty}\left|\lambda_{n+1}-\lambda_{n}\right|<\infty$, and $\sum_{n=0}^{\infty}\left|\mu_{n+1}-\mu_{n}\right|<\infty$.

By the careful analysis of the proof of Theorem 19, we can obtain the following result. Because its proof is much simpler than that of Theorem 19, we omit its proof.

Theorem 21. Let $C$ be a nonempty closed convex subset of a uniformly convex and 2-uniformly smooth Banach space $X$ such that $C \pm C \subset C$. Let $\Pi_{C}$ be a sunny nonexpansive retraction from $X$ onto $C$. Let the mapping $B_{i}: C \rightarrow X$ be $\alpha_{i}$-inverse-strongly accretive for $i=1,2$. Let $\left\{S_{n}\right\}_{n=0}^{\infty}$ be an infinite family of nonexpansive mappings of $C$ into itself such that $\Delta=\bigcap_{i=0}^{\infty} \operatorname{Fix}\left(S_{i}\right) \cap \Omega \neq \emptyset$, where $\Omega$ is the fixed point set of the mapping $G=\Pi_{C}\left(I-\mu_{1} B_{1}\right) \Pi_{C}\left(I-\mu_{2} B_{2}\right)$ with $0<\mu_{i}<\alpha_{i} / \kappa^{2}$ for $i=1,2$. Let $f: C \rightarrow C$ be a fixed contractive map with coefficient $\beta \in(0,1)$, let $F: C \rightarrow C$ be $\alpha$-strongly accretive and $\lambda$-strictly pseudocontractive with $\alpha+\lambda>1$, and let $A: C \rightarrow C$ be a $\bar{\gamma}$-strongly positive linear bounded operator with $0<\bar{\gamma}-\beta \leq 1$. Given sequences $\left\{\lambda_{n}\right\}_{n=0}^{\infty}$ in $[0,1]$ and $\left\{\alpha_{n}\right\}_{n=0}^{\infty},\left\{\beta_{n}\right\}_{n=0}^{\infty}$ in $(0,1]$, suppose that there hold the following conditions:

(i) $\lim _{n \rightarrow \infty} \beta_{n}=0$ and $\sum_{n=0}^{\infty} \beta_{n}=\infty$;

(ii) $\lim _{n \rightarrow \infty} \lambda_{n} / \beta_{n}=0$ and $\sum_{n=0}^{\infty}\left|\lambda_{n+1}-\lambda_{n}\right|<\infty$;

(iii) $\left\{\alpha_{n}\right\} \subset[a, b]$ for some $a, b \in(0,1)$;

(iv) $\sum_{n=0}^{\infty}\left|\alpha_{n+1}-\alpha_{n}\right|<\infty$ and $\sum_{n=0}^{\infty}\left|\beta_{n+1}-\beta_{n}\right|<\infty$.

Assume that $\sum_{n=0}^{\infty} \sup _{x \in D}\left\|S_{n+1} x-S_{n} x\right\|<\infty$ for any bounded subset $D$ of $C$ and let $S$ be a mapping of $C$ into itself defined by $S x=\lim _{n \rightarrow \infty} S_{n} x$ for all $x \in C$ and suppose that $\operatorname{Fix}(S)=$ $\bigcap_{n=0}^{\infty} \operatorname{Fix}\left(S_{n}\right)$. Then, for any given point $x_{0} \in C$, the sequence $\left\{x_{n}\right\}$ generated by

$$
\begin{gathered}
y_{n}=\alpha_{n} x_{n}+\left(1-\alpha_{n}\right) G\left(S_{n} x_{n}\right), \\
x_{n+1}=\beta_{n} f\left(x_{n}\right)+\left(I-\beta_{n} A\right)\left[y_{n}-\lambda_{n} F\left(y_{n}\right)\right], \quad \forall n \geq 0,
\end{gathered}
$$

converges strongly to $p \in \Delta$, which is the unique solution in $\Delta$ to the VIP (85).

Remark 22. Theorems 19 and 21 improve, extend, supplement and develop Cai and $\mathrm{Bu}$ [10, Theorem 3.1] and Cai and $\mathrm{Bu}$ [9, Theorems 3.1] in the following aspects.

(i) The GSVI (13) with hierarchical fixed point problem constraint for a countable family of nonexpansive mappings is more general and more subtle than every problem in Cai and $\mathrm{Bu}[10$, Theorems 3.1] and $\mathrm{Cai}$ and $\mathrm{Bu}[9$, Theorem 3.1] 
because our problem is to find a point $p \in \Delta=\bigcap_{n} \operatorname{Fix}\left(S_{n}\right) \cap \Omega$, which is the unique solution in $\Delta$ to the VIP:

$$
\langle(A-f) p, J(p-u)\rangle \leq 0, \quad \forall u \in \Delta .
$$

(ii) The iterative scheme in [10, Theorem 3.1] is extended to develop the iterative schemes in Theorems 19 and 21 by virtue of hybrid steepest-descent method. The iterative schemes in Theorems 19 and 21 are more advantageous and more flexible than the iterative scheme of [9, Theorem 3.1] because the iterative scheme of [9, Theorem 3.1] is implicit and our iterative schemes involve solving two problems: the GSVI (13) and the fixed point problem of a countable family of nonexpansive mappings $\left\{S_{n}\right\}$.

(iii) The iterative schemes in Theorems 19 and 21 are very different from everyone in both [10, Theorem 3.1] and [9, Theorem 3.1] because our iterative schemes involve hybrid steepest-descent method (namely, we add a strongly accretive and strictly pseudocontractive mapping $F$ in our iterative schemes) and because the mappings $G$ and $S_{n}$ in [10, Theorem 3.1] and the mapping $S_{n}$ in [9, Theorem 3.1] are replaced by the same composite mapping $G \circ S_{n}$ in the iterative schemes of Theorems 19 and 21.

(iv) Cai and Bu's proof in [10, Theorem 3.1] depends on the argument techniques in [20], the inequality in 2uniformly smooth Banach spaces (see Lemma 1), and the inequality in smooth and uniform convex Banach spaces (see Proposition 2). Because the composite mapping $G \circ S_{n}$ appears in the iterative schemes in Theorems 19 and 21, the proof of Theorems 19 and 21 depends on the argument techniques in [20], the inequality in 2-uniformly smooth Banach spaces (see Lemma 1), the inequality in smooth and uniform convex Banach spaces (see Proposition 2), and the properties of the strongly positive linear bounded operator (see Lemmas 15), the Banach limit (see Lemma 5), and the strongly accretive and strictly pseudocontractive mapping (see Lemma 7).

Remark 23. Theorems 19 and 21 extend and improve Theorem 16 of Yao et al. [21] to a great extent in the following aspects:

(i) the $u$ is replaced by a fixed contractive mapping;

(ii) one continuous pseudocontractive mapping (including nonexpansive mapping) is replaced by a countable family of nonexpansive mappings;

(iii) we add a strongly positive linear bounded operator $A$ and a strongly accretive and strictly pseudocontractive mapping $F$ in our iterative algorithms.

\section{Conflict of Interests}

The authors declare that there is no conflict of interests regarding the publication of this paper.

\section{Acknowledgment}

This article was funded by the Deanship of Scientific Research (DSR), King Abdulaziz University, Jeddah. The authors, therefore, acknowledge with thanks DSR for the technical and financial support. This research was partially supported to first author by the National Science Foundation of China (11071169), Innovation Program of Shanghai Municipal Education Commission (09ZZ133), and Ph.D. Program Foundation of Ministry of Education of China (20123127110002). Finally, the authors thank the referees for their valuable comments and suggestions for improvement of the paper.

\section{References}

[1] H. Iiduka and W. Takahashi, "Strong convergence theorems for nonexpansive mappings and inverse-strongly monotone mappings," Nonlinear Analysis A, vol. 61, no. 3, pp. 341-350, 2005.

[2] L.-C. Ceng, Q. H. Ansari, and J.-C. Yao, "An extragradient method for solving split feasibility and fixed point problems," Computers \& Mathematics with Applications, vol. 64, no. 4, pp. 633-642, 2012.

[3] L.-C. Ceng, Q. H. Ansari, and J.-C. Yao, "Relaxed extragradient methods for finding minimum-norm solutions of the split feasibility problem," Nonlinear Analysis A, vol. 75, no. 4, pp. 2116-2125, 2012.

[4] L.-C. Ceng, Q. H. Ansari, and J.-C. Yao, "Relaxed extragradient iterative methods for variational inequalities," Applied Mathematics and Computation, vol. 218, no. 3, pp. 1112-1123, 2011.

[5] L.-C. Ceng, Q. H. Ansari, and J.-C. Yao, "Mann-type steepestdescent and modified hybrid steepest-descent methods for variational inequalities in Banach spaces," Numerical Functional Analysis and Optimization, vol. 29, no. 9-10, pp. 987-1033, 2008.

[6] W. Takahashi and M. Toyoda, "Weak convergence theorems for nonexpansive mappings and monotone mappings," Journal of Optimization Theory and Applications, vol. 118, no. 2, pp. 417428, 2003.

[7] H. Iiduka, W. Takahashi, and M. Toyoda, "Approximation of solutions of variational inequalities for monotone mappings," Panamerican Mathematical Journal, vol. 14, no. 2, pp. 49-61, 2004.

[8] Y. Takahashi, K. Hashimoto, and M. Kato, "On sharp uniform convexity, smoothness, and strong type, cotype inequalities," Journal of Nonlinear and Convex Analysis, vol. 3, no. 2, pp. 267281, 2002.

[9] G. Cai and S. Bu, "Approximation of common fixed points of a countable family of continuous pseudocontractions in a uniformly smooth Banach space," Applied Mathematics Letters, vol. 24, no. 12, pp. 1998-2004, 2011.

[10] G. Cai and S. Bu, "Convergence analysis for variational inequality problems and fixed point problems in 2-uniformly smooth and uniformly convex Banach spaces," Mathematical and Computer Modelling, vol. 55, no. 3-4, pp. 538-546, 2012.

[11] H. K. Xu, "Inequalities in Banach spaces with applications," Nonlinear Analysis A, vol. 16, no. 12, pp. 1127-1138, 1991.

[12] S. Kamimura and W. Takahashi, "Strong convergence of a proximal-type algorithm in a Banach space," SIAM Journal on Optimization, vol. 13, no. 3, pp. 938-945, 2002.

[13] H. K. Xu and T. H. Kim, "Convergence of hybrid steepestdescent methods for variational inequalities," Journal of Optimization Theory and Applications, vol. 119, no. 1, pp. 185-201, 2003.

[14] W. Takahashi, Nonlinear Functional Analysis-Fixed Point Theory and Its Applications, Yokohama Publishers, Yokohama, Japan, 2000, Japanese. 
[15] S. Reich, "Weak convergence theorems for nonexpansive mappings in Banach spaces," Journal of Mathematical Analysis and Applications, vol. 67, no. 2, pp. 274-276, 1979.

[16] R. E. Bruck, Jr., "Properties of fixed-point sets of nonexpansive mappings in Banach spaces," Transactions of the American Mathematical Society, vol. 179, pp. 251-262, 1973.

[17] K. Aoyama, Y. Kimura, W. Takahashi, and M. Toyoda, "Approximation of common fixed points of a countable family of nonexpansive mappings in a Banach space," Nonlinear Analysis A, vol. 67, no. 8, pp. 2350-2360, 2007.

[18] L.-C. Ceng and J.-C. Yao, "An extragradient-like approximation method for variational inequality problems and fixed point problems," Applied Mathematics and Computation, vol. 190, no. 1, pp. 205-215, 2007.

[19] Y. Censor, A. Gibali, and S. Reich, "Two extensions of Korpelevich's extragradient method for solving the variational inequality problem in Euclidean space," Technical Report, 2010.

[20] L.-C. Ceng, C.-y. Wang, and J.-C. Yao, "Strong convergence theorems by a relaxed extragradient method for a general system of variational inequalities," Mathematical Methods of Operations Research, vol. 67, no. 3, pp. 375-390, 2008.

[21] Y. Yao, Y.-C. Liou, and R. Chen, "Strong convergence of an iterative algorithm for pseudocontractive mapping in Banach spaces," Nonlinear Analysis A, vol. 67, no. 12, pp. 3311-3317, 2007. 


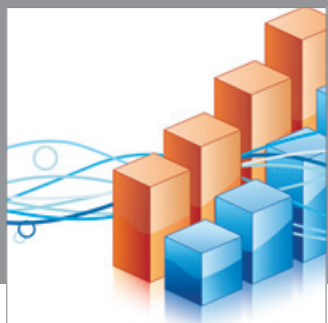

Advances in

Operations Research

mansans

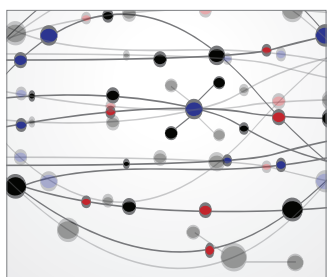

The Scientific World Journal
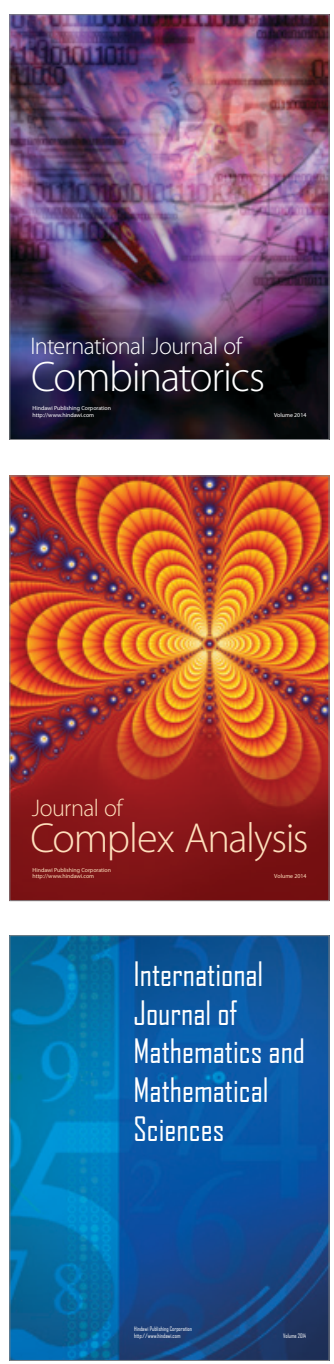
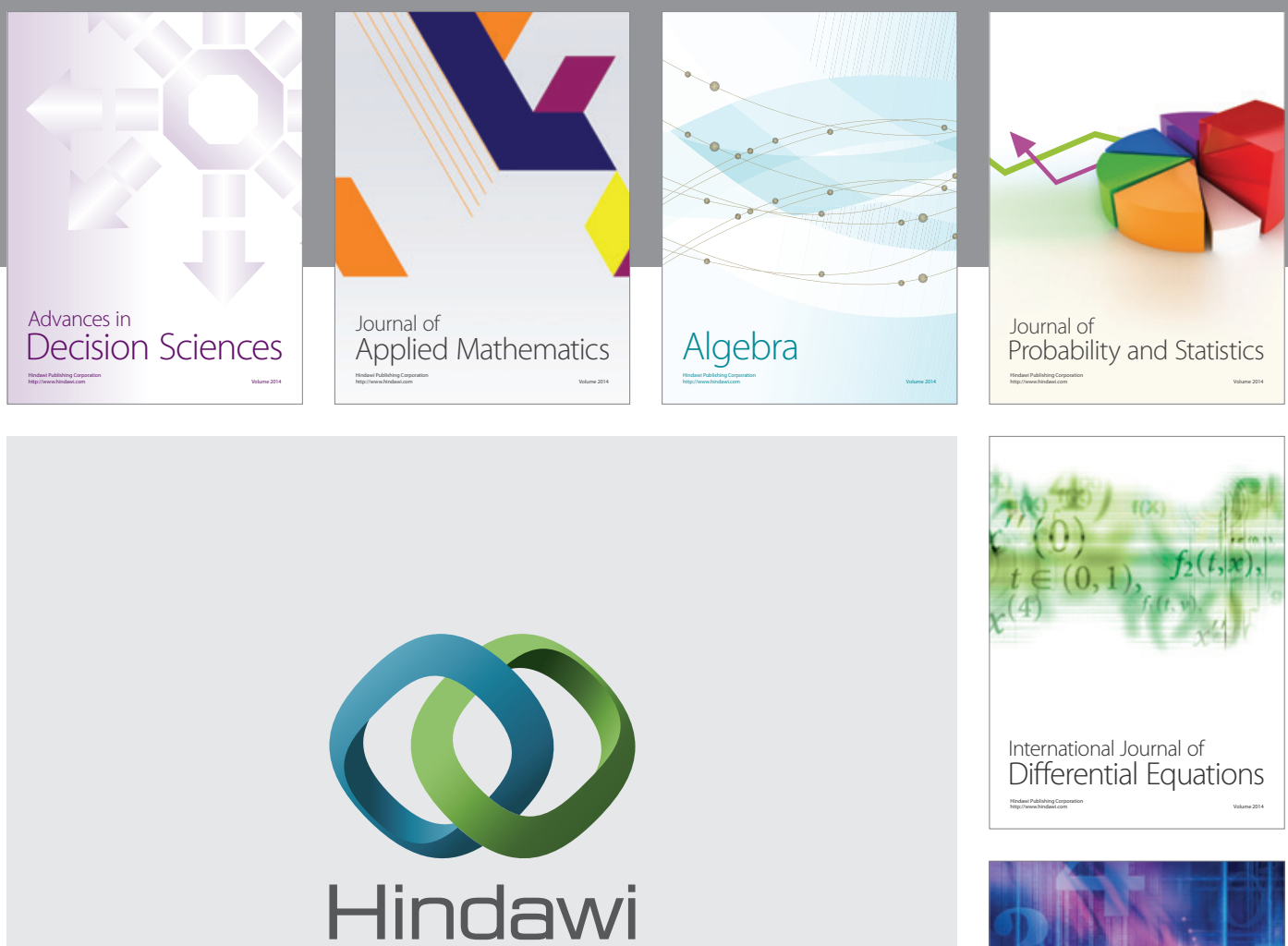

Submit your manuscripts at http://www.hindawi.com
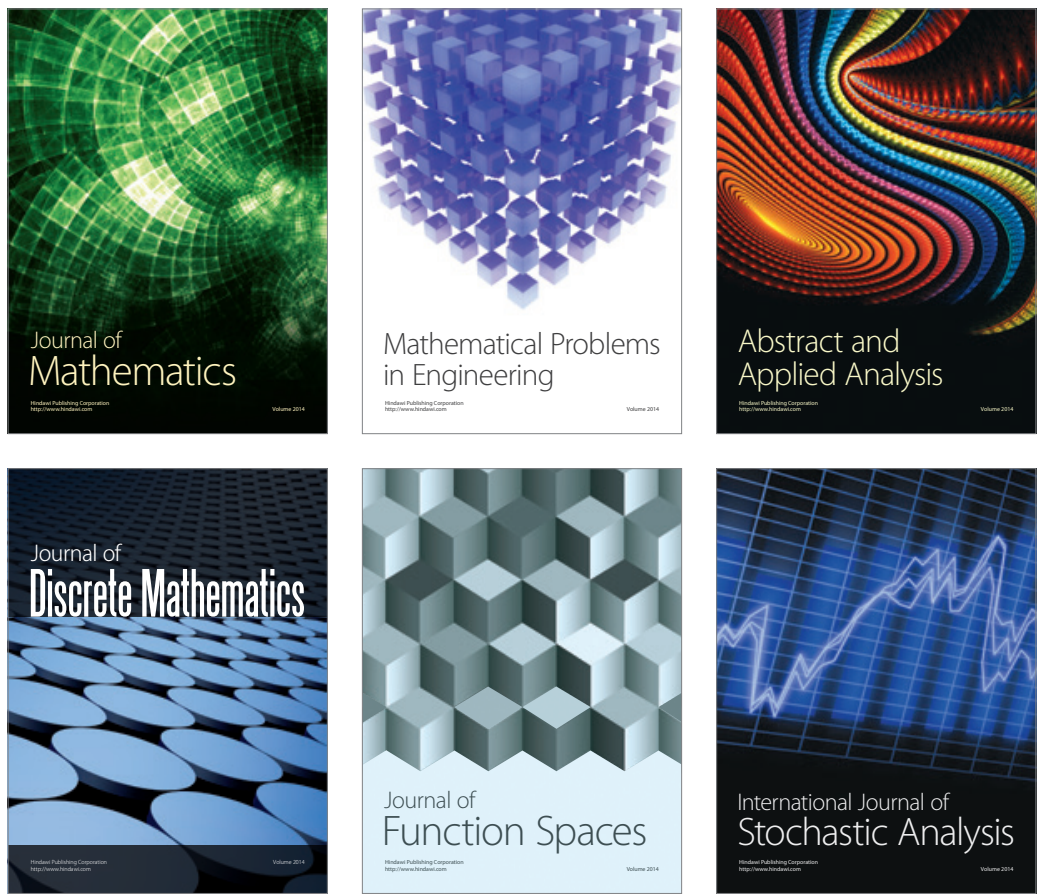

Journal of

Function Spaces

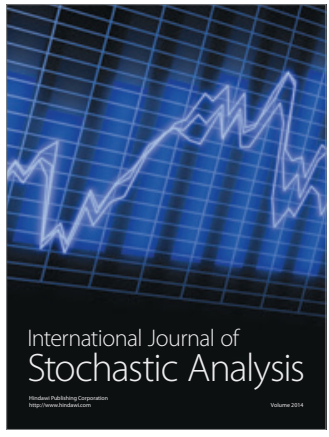

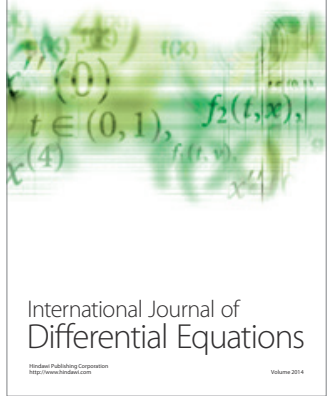
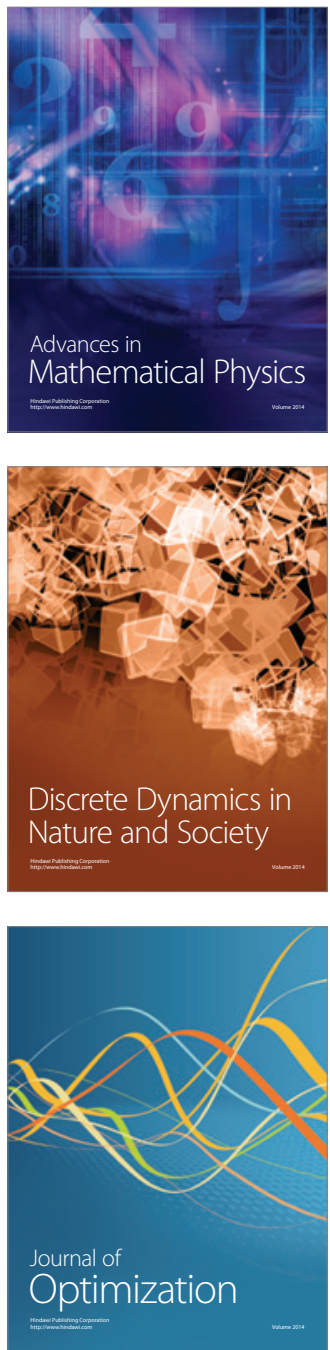